\title{
Plantas invasoras en el Parque Nacional Torres del Paine (Magallanes, Chile): estado del arte, distribución post-fuego e implicancias en restauración ecológica
}

Invasive plants in Torres del Paine National Park (Magallanes, Chile): current knowledge, post-fire distribution and implications for ecological restoration

Osvaldo J. Vidal ${ }^{1}$, Mauricio Aguayo ${ }^{2}$, Roberto Niculcar ${ }^{3}$, Nelson Bahamonde ${ }^{4}$, Sergio Radic, Cristina San Martín ${ }^{6}$, Alejandro Kusch ${ }^{7}$, Javier Latorre ${ }^{1}$ \& Jorge Félez ${ }^{2}$

\section{Resumen}

El Parque Nacional Torres del Paine (TDP) es un área donde las plantas invasoras son una amenaza creciente para la conservación. El aumento de turistas ha propiciado durante los últimos años un incremento de invasoras en sitios donde el turismo tiene impacto directo (e.g. áreas de camping, senderos). En ocasiones, turistas negligentes han provocado mega-incendios que han favorecido la dispersión de estas plantas. En este trabajo se revisa la información concerniente a las plantas exóticas en TDP, y se utiliza un marco conceptual para abordar esta problemática, que incluye conceptos jerárquicos de invasibilidad. Se presentan resultados para dos experiencias en donde este marco conceptual tiene aplicación: a) una prospección en que se evalúa la distribución y abundancia de exóticas post-incendio; b) un experimento de campo donde se evalúa el efecto del control de invasoras en la supervivencia de plántulas de lenga (Nothofagus pumilio). Además se entregan antecedentes que permiten calcular el costo de la operación de control. La investigación sobre plantas exóticas en TDP incluye listados de especies que categorizan a priori las invasoras, careciendo de información ecológica de campo. Se detectó la presencia de 34 especies de plantas exóticas en sitios incendiados, algunas de ellas con gran potencial de invasión postincendio. La supervivencia de plántulas de Lenga es mayor cuando se han controlado las malezas mediante remoción mecánica, posiblemente debido a la facilitación inducida. Los costos de control de especies de malezas mediante remoción mecánica pueden fluctuar desde CLP\$ 700.000 a \$1.400.000 para el control de 1 hectárea. Este estudio resalta la necesidad de considerar a las especies invasoras como un fenómeno contextodependiente, que puede o no persistir dependiendo del tipo de perturbación que facilite su dispersión, grados de severidad, tiempo post-perturbación y resiliencia ecosistémica.

\section{Palabras clave:}

Ecosistemas subantárticos, invasión post-incendio, Rumex acetosella, Plantago lanceolata, Fuego, control de plantas invasoras.

\section{Abstract}

Torres del Paine National Park (TDP) is an area in which invasive plants are a growing threat for

\footnotetext{
1 Laboratorio de Botánica "Edmundo Pisano", Instituto de la Patagonia, Universidad de Magallanes. osvaldo.vidal@umag.cl $\square$

2 Laboratorio de Geomática, Centro EULA, Universidad de Concepción.

3 Laboratorio de Conservación de Germoplasma, Servicio Agrícola \& Ganadero Magallanes.

4 ONG AMA Torres del Paine.

5 Laboratorio de Suelos, Facultad de Ciencias, Universidad de Magallanes.

6 Instituto de Ciencias Ambientales \& Evolutivas, Universidad Austral de Chile.

$7 \quad$ Wildlife Conservation Society, Punta Arenas.
} 
the biodiversity conservation. The growing number of visitors in TDP has allowed in the last years the increase of invasive species in places where tourism has a direct impact (e.g. camping areas, trails). In other occasions negligent tourists have set mega-fires that stimulate the dispersion of this plants. In this work we review the information concerning on alien plant species in TDP and we use a conceptual framework widely accepted to address this problematic. We present the results of two field experiences in which this conceptual framework has been applied: a) a prospection where the distribution and the abundance of postfire exotics are evaluated; b) a field experiment in which we evaluated the effect of invasive plant control on Lenga seedlings. These seedlings were planted with reforestation purposes. Furthermore we give the background that allows the calculation of the monetary costs of the weed control. The investigation of alien plant species includes checklists that categorize a priori the invasive species, lacking of field ecologic information. We recorded the presence of 34 exotic plant species in burned places, some of them having a great postfire invasibility potential. Meanwhile in the sites where reforestation occurs the survival of lenga beech seedlings is higher when the weeds has been controled by mechanical removal, likely by the facilitation induced by control. The costs of weed control fluctuate from CLP\$ 700.000 to 1.400 .000 per hectare (USD\$ 1.135 to 2.270). This study highlights the need to consider the invasive plant species as a context-dependent phenomenon that may or may not persists, depending on the type of disturbance that facilitates their dispersion, severity, post-disturbance time and ecosistemic resilience.

\section{Key words:}

Subantartic ecosystems, Post-fire invasion, Rumex acetosella, Plantago lanceolata, Fire, Control of invasive plants.

\section{INTRODUCCIÓN}

El Parque Nacional Torres del Paine es una de las áreas protegidas más importantes en Chile, debido principalmente a la belleza escénica del macizo montañoso al cual debe su nombre. Este escenario constituye la postal por antonomasia de
Chile, y convoca un número creciente de turistas. La Cordillera Paine se alza abruptamente hasta poco mas de los $3.000 \mathrm{~m}$ de elevación, que junto al Glaciar Grey como componente del Campo de hielo Patagónico-Sur, generan un fuerte gradiente climático que proporciona una alta heterogeneidad de paisajes vegetacionales (Pisano, 1974) (Fig. 1). Estos incluyen bosques perennifolios, bosques caducifolios, matorrales preandinos, turberas y la llana estepa patagónica (Pisano, 1974; Clausen et al. 2006; Vidal \& Bauk, 2014). Esta heterogeneidad paisajística concentra una alta diversidad de especies de plantas vasculares (Arroyo et al. 1992; Vidal, 2007) y de fauna característica de los ecosistemas templados australes de Chile y Argentina (e.g. Ortega \& Franklin, 1988; Iriarte et al. 1991). Se estima que el área concentra mas de 450 especies de plantas, y mas de 130 especies de aves (Couve \& Vidal, 2007; Vidal, 2007), incluyendo la mayor riqueza de aves rapaces reportada para un área protegida en Chile (Jaksic et al. 2002).

El contexto escénico y la biodiversidad que alberga el Parque Nacional Torres del Paine genera un atractivo singular que se ha traducido en un incremento masivo de turistas durante los últimos decenios. Durante las últimas temporadas, han ingresado mas de 190.000 turistas cada año (Conaf, estadísticas no publicadas), lo que ha significado en ocasiones puntuales, un turismo negligente que ha redundado en serias consecuencias para la biodiversidad local. Tres eventos de incendios producidos por turistas han cobrado particular importancia por la relevancia ecológica de los mismos, significando la destrucción de vastas superficies con ecosistemas nativos (Navarro-Cerrillo et al. 2008) y transformación de los mismos hacia ecosistemas seminaturales, de origen antropogénico, y con dominancia de especies colonizadoras post-incendio (Vidal $\&$ Bau, 2014). Estos tres mega-incendios han destruido poco mas del $20 \%$ de la superficie del Parque Nacional (unas 47.000 hectáreas) (Vidal, 2012a), y muchos de sus efectos ecológicos aún son desconocidos. Adicionalmente, previo a que el área fuese declarada protegida por el Estado de Chile, ésta estuvo sujeta a una fuerte influencia antropogénica por actividades ganaderas (Martinic, 1985; Roig et al. 1985).

La influencia humana en el área de estudio, 


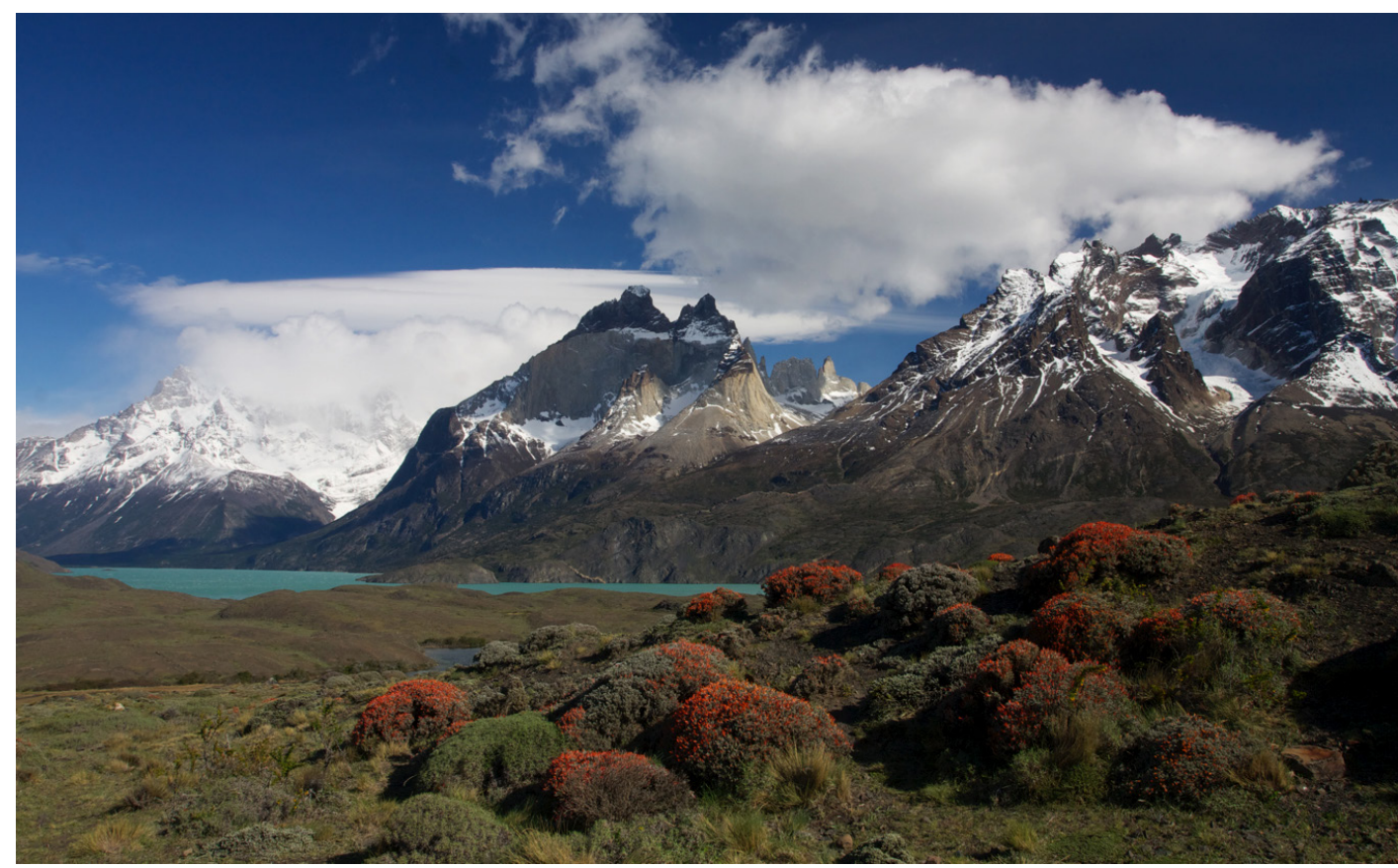

Fig. 1. Fisonomía de la Cordillera Paine, con los "Cuernos del Paine" y el monte "Almirante Nieto" dominando la posición central y lateral derecha del cuadro. La abrupta elevación del macizo, alcanzando poco mas de $3.050 \mathrm{~m}$ de elevación, captura los vientos húmedos predominantes del noroeste, generando un drástico gradiente eco-climático en el que es posible observar una diversidad representativa ecosistemas Subantárticos, desde bosques perennifolios, bosques caducifolios, matorrales preandinos y estepa Patagónica (Fotografía Claudio Vidal).

sea durante los tiempos de colonización, o más recientemente por turistas, ha repercutido en un incremento en la frecuencia de incendios antrópicos en la región de estudio (Huber \& Markgraf, 2003). De acuerdo a investigaciones palinológicas desarrolladas, los incendios son eventos que han ocurrido en oleadas recurrentes durante los últimos 10.000 años en el área de estudio (VillaMartínez \& Moreno, 2007), como consecuencia de cambios climáticos que a escalas de siglos significaron fases de climas secos y calurosos con una duración aproximada de doscientos años, las que se correspondieron con incrementos de incendios (Moreno et al. 2014). La vegetación dominante habría sido capaz de recuperarse luego de terminada la fase calurosa y seca (Moreno et al. 2014). En la actualidad el incremento en la frecuencia de los mega-incendios y la permanente presencia antrópica plantean la interrogante sobre la capacidad de resiliencia de los ecosistemas en Torres del Paine. Además, estos ecosistemas han incorporado numerosas especies exóticas, que introducidas durante los tiempos de colonización o más recientemente por turistas (Vidal, 2005; Domínguez et al. 2006; Vidal \& Reif, 2011), se han naturalizado en vastas áreas dificultando los procesos naturales de regeneración de especies arbóreas, como la lenga (Nothofagus pumilio) (Vidal \& Reif, 2011).

Las invasiones biológicas representan un problema cuya relevancia se ha ido incrementando durante el últimos decenios dada la importancia ecológica que significa en disciplinas como conservación de la biodiversidad y restauración ecológica (Mooney \& Hobbs 2000). Los problemas ambientales que implican las invasiones biológicas afectan los diferentes niveles de organización de la biodiversidad (sensu Noss, 1990) y fluctúan desde cambios a escala de flujo genético interpoblacional (Chornesky \& Randall, 2003), hasta disrupciones a escala de paisaje (Gelbard \& Belnap, 2003). Sin embargo, el enfoque otorgado a las especies exóticas en áreas protegidas en Chile es muy amplio, y en la mayoría de las ocasiones el estatus de invasión de las especies exóticas es desconocido. Más aún, las especies catalogadas como invasoras, vale decir, 
aquellas exóticas que son capaces de reproducirse en gran número y fuera de las zonas de cultivo o de influencia antrópica directa (sensu Pyšek et al. 2004), pueden representar un fenómeno temporal mediado por el tipo de perturbación que promueve su expansión, edad de la perturbación y/o tipo de ecosistema invadido (i.e. resiliencia del ecosistema).

Parece pertinente preguntarse entonces, desde el punto de vista de las invasiones por plantas exóticas: ¿Cuáles son las consecuencias ecológicas de los incendios en ecosistemas Subantárticos de Torres del Paine? ¿Son estas consecuencias de largo plazo? y de existir efectos ecológicos duraderos, ¿Pueden idearse mecanismos artificiales de restauración para estos ecosistemas? Para responder al menos parcialmente estas preguntas, en primer lugar se desarrolla una revisión bibliográfica sobre los trabajos publicados en Torres del Paine y localidades adyacentes, referentes a flora, vegetación e impacto antrópico por fuego. Seguidamente, se muestran resultados inéditos para dos experiencias de campo en Torres del Paine: a) una prospección desarrollada en el área afectada por el último mega-incendio del 2011-2012 (17.054 hectáreas) cuya finalidad fue registrar la riqueza y cobertura florística en sitios con diferentes severidades de incendio; b) un experimento de reforestación y control mecánico de especies de plantas invasoras cuya finalidad, a posteriori fue evaluar el efecto de control de estas especies sobre la supervivencia de plántulas de lenga. Simultáneamente, se integran estas experiencias en el marco conceptual propuesto por Pyšek et al. (2004), quienes categorizan a las especies esxóticas en diferentes grados de invasibilidad. Este marco conceptual es ampliamente empleado en la literatura sobre invasiones biológicas, cuyo potencial en manejo de especies invasoras se aplica directamente en las experiencias de campo desarrolladas en éste estudio. Por consiguiente los objetivos de este estudio fueron: a) Compilar la información sobre plantas exóticas en Torres del Paine; b) registrar las especies exóticas en sitios recientemente afectados por el fuego en Torres del Paine; b) evaluar el efecto del control de invasoras en la supervivencia de plántulas de lenga y; c) integrar la información de campo en el esquema conceptual propuesto por Pyšek et al. (2004) para la clasificación de las plantas exóticas.

\section{MATERIALES Y MÉTODOS}

\section{Revisión bibliográfica}

Se llevó a cabo una revisión bibliográfica de la literatura ecológica publicada sobre el Parque Nacional Torres del Paine. Labúsqueda, realizada principalmente online, incluyó palabras claves mediante operadores boleanos de la base de datos ampliada del Web of Science, esto es, la base de datos principal Web of Science Core Collection (WOS), además de la base de datos del SciELO Citation Index. Las palabras claves incluyeron conceptos como: "Torres del Paine", "Flora", "Vegetation", "Nothofagus", "Fire", "Exotic Plant Species", e "Invasive plants" (operadores AND y $\mathrm{OR})$. Dado que el número de artículos resultantes de la búsqueda combinada fue muy bajo (8 publicaciones), se eliminó el concepto "Torres del Paine" y reemplazó por "Chile" y "Patagonia" para ampliar la búsqueda hacia ecosistemas subantárticos, cuya información pudiera tener una potencial aplicación en el área de estudio. Además, se revisó el catálogo completo de la revista Anales del Instituto de la Patagonia (ausente del WOS), para localizar contribuciones adicionales de estudios realizados en Torres del Paine y/o ecosistemas subantárticos afines. Finalmente, la búsqueda fue completada mediante la inclusión de numerosa literatura gris (sensu García, 1998), no convencional (e.g. informes técnicos, tesis), aportada por agencias gubernamentales (e.g. Corporación Nacional Forestal, Ministerio del Medio Ambiente, Universidad de Magallanes) y no gubernamentales (e.g. ONG AMA Torres del Paine, Universidad Austral de Chile, Universidad de Concepción, Universidad de Chile, Instituto de Ecología \& Biodiversidad), que incluían estudios realizados en el área de interés.

\section{Procedimiento de campo para \\ el registro de especies exóticas}

El área de estudio correspondió a la superficie incendiada en el año 2011 en Torres del Paine. La aproximación del diseño de muestreo priorizó la escala de micrositio, dado que es presumible que los cambios en las condiciones de micrositio como 

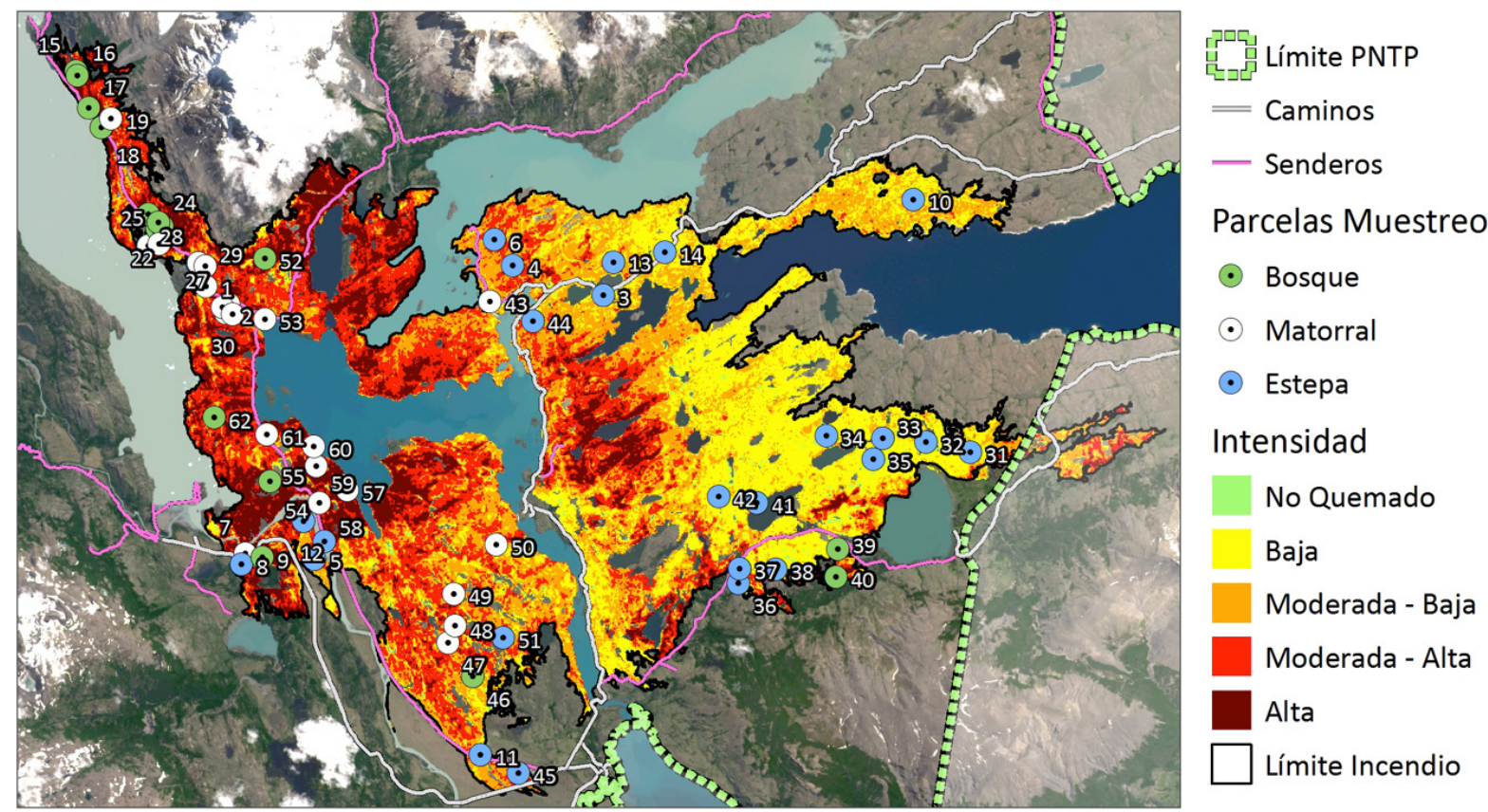

Fig. 2. Mapa de severidades para el último mega-incendio ocurrido en 2011-2012 en Torres del Paine, mostrando la distribución de las unidades de muestreo para el registro de plantas vasculares en sitios afectados por diferentes severidades de incendio. Los puntos, además, son clasificados de acuerdo a la vegetación predominante pre-incendio (e.g. estepa, matorral, bosque).

consecuencia del fuego (i.e. variables explicatorias; e.g. severidad de incendio) tengan una mayor influencia sobre el desarrollo post-fuego de la vegetación. Por otra parte, la detección de especies menos conspicuas (e.g. flores no vistosas, plantas diminutas) es mayor cuando el área a muestrear es más pequeña (Mueller-Dombois \& Ellenberg, 1974). Por esto, la unidad de muestreo consistió en una parcela de vegetación (i.e. relevamiento florístico) de $25 \mathrm{~m}^{2}$ (cuadrante de $5 \times 5 \mathrm{~m}$ ), que revela un gran porcentaje de la riqueza florística de las unidades vegetacionales (i.e. comunidades vegetales). La selección de los sitios de estudio fue estratificada, basada en el mapa de severidad del incendio desarrollada previamente (Ministerio del Medio Ambiente, 2014) y las coberturas de la vegetación dominante pre-incendio (e.g. bosque, matorral, estepa sensu Pisano, 1974). Se seleccionaron micrositios con severidades baja, moderada-baja, moderada-alta, y alta en esfuerzos de muestreo balanceado en cada uno de los estratos (en total 62 unidades de muestreo) (Fig. 2 ), distribuido aleatoriamente según la superficie ocupada por las coberturas vegetacionales existentes pre-incendio. En cada micrositio se estableció la unidad de muestreo para evaluar variables respuesta referidas a la composición florística (presencia de la diversidad de especies de plantas vasculares, cobertura de la vegetación en el sotobosque) (Mueller-Dombois \& Ellenberg, 1974; Vidal, 2007). En cada parcela de vegetación se registró cada una de las especies de plantas vasculares, además de registrar la cobertura relativa de las especies en escalas de porcentajes como sigue: $+=$ coberturas menores al $1 \% ; 1=$ entre $1 \%$ y $5 \%$ de cobertura; $2=$ entre $5 \%$ y $25 \%$ de cobertura; $3=$ entre $26 \%$ y $50 \%$ de cobertura; $4=$ entre $51 \%$ y $75 \%$ de cobertura; $5=$ entre $76 \%$ y $100 \%$ de cobertura (Mueller-Dombois \& Ellenberg, 1974). Cuando la determinación a nivel taxonómico de especie no fue posible, se colectaron ejemplares de las plantas para su determinación en laboratorio de acuerdo a literatura taxonómica morfológica (e.g. Moore, 1983; Rodríguez \& Quezada, 2003; Marticorena, 2006; Vidal, 2007; Vidal et al. 2012).

Se determinó el origen fitogeográfico de las especies de plantas vasculares de acuerdo a literatura taxonómica (e.g. Moore, 1983; Dollenz \& Ivanovic, 1986; Henríquez et al. 1995; Domínguez et al. 
2006) y se filtró una matriz de especies de plantas exóticas por unidad de muestreo. Esta matriz fue tabulada en orden decreciente de acuerdo a las abundancias y frecuencias relativas de las especies para jerarquizarlas según el "valor de importancia" de Wikum (Wikum \& Shanholtzer, 1978). Además, los sitios de estudio fueron asignados a las diferentes categorías de acuerdo al tipo de vegetación pre-incendio (bosques, matorrales, estepas) y a los niveles de severidad (bajo, moderado-bajo, moderado-alto, alto) y sometidos a análisis de especies indicadoras (Dufrene \& Legendre, 1997; De Cáceres et al . 2010) para evaluar la consistencia entre los resultados arrojados por el valor de importancia de Wikum \& Shanholtzer (1978) y los valores arrojados por el método de especies indicadoras de Dufrene \& Legendre (1997). Así, se determinaron las 10 especies de plantas exóticas más frecuentes y abundantes a través de todos los sitios de estudio, grados de severidad del fuego y/o vegetación pre-incendio. El número de especies de mayor importancia como invasoras (sensu Pyšek et al. 2004) fue seleccionado arbitrariamente (10 especies), con el objeto de rankearlas de acuerdo a los resultados de campo.

\section{Experiencias de campo sobre control de invasoras}

Las acciones de control de plantas vasculares exóticas fueron llevadas a cabo experimentalmente en el sector del Lago Grey en Torres del Paine, particularmente el sitio denominado "Cuesta Margarita". En este sitio, la ONG AMA Torres del Paine implementó parte de su "Plan de Restauración de Bosques Incendiados" en mayo del 2013 plantando 2.346 plántulas de lenga (Nothofagus pumilio) de un total de 20.000 plantadas en otros dos sitios (Vidal \& Bauk, 2014). Dichas plantaciones fueron ejecutadas mediante la técnica de nucleación, es decir, mediante grupos de plántulas plantadas en altas densidades, distanciadas entre sí irregularmente entre 25-40 cm $( \pm 60$ plantas en un área irregular de aproximadamente 3-6 $\mathrm{m}^{2}$, equivalentes a densidades de \pm 150.000 190.000 plántulas por hectárea), técnica que imita las densidades de regeneración natural observadas en otros estudios (Cavieres \& Fajardo, 2005). La localización de dichos núcleos fue determinada mediante la presencia de "legados biológicos", esto es, estructuras orgánicas remanentes de incendios como arbustos, troncos grandes muertos en pie o en el suelo y/o árboles vivos aislados o bordes de fragmentos boscosos aislados (Franklin, 1990). Estos legados biológicos pueden ayudar a incrementar la sobrevivencia de las plántulas mediante protección por semi-sombra, captura y retención de humedad, protección contra el ramoneo, entre otros factores facilitadores.

Esta configuración de plantación permitió el diseño de un experimento de terreno para evaluar el efecto del control de plantas invasoras sobre la supervivencia de plántulas de lenga. Las plantaciones de Lenga del Plan AMA Torres del Paine en el sitio de estudio fueron ejecutadas en Otoño del año 2013 por un grupo de voluntarios, quienes establecieron 40 núcleos con alrededor de 58 plantas cada uno (2.346 plantas en total) en una superficie de 1 hectárea (Vidal \& Bauk, 2014). Para evaluar la supervivencia de las plántulas postinvierno, estos núcleos fueron inspeccionados por el equipo de la ONG en octubre del 2013, comprobando que el $97 \%$ de las plántulas habían sobrevivido al proceso de plantación y a la dormancia de invierno (Vidal \& Bauk, 2014). Así, estos núcleos se convirtieron en sitios de interés para establecer la primera experiencia de control de plantas exóticas en micro-sitios con restauración activa en bosques incendiados de Torres del Paine.

El experimento consistió en remover plantas invasoras en 10 de los 40 núcleos establecidos, los cuales contenían cerca de 55 plántulas de lenga cada uno (542 plantas en total) (Fig. 3). La actividad de remoción se realizó el 12 de enero de 2013 por un equipo de 6 personas. Además, se establecieron 10 núcleos adicionales como control con la finalidad de evaluar la supervivencia de plántulas de lenga en sitios sin remoción de plantas invasoras. Estos núcleos contenían 546 plántulas de lenga. La actividad de remoción de plantas invasoras consistió en la extracción manual de todas las especies presentes en los núcleos de restauración. Tres meses más tarde (Abril de 2013) se procedió a la contabilización de los individuos de lenga tanto en los núcleos intervenidos como en los núcleos control. Se evaluaron las diferencias entre los núcleos tratados y no tratados en base al porcentaje de supervivencia obtenido para ambas 
situaciones. Las diferencias entre medianas fueron comparadas usando la prueba U-Mann Whitney (Sokal \& Rohlf, 1981).

\section{Marco conceptual para el manejo de plantas invasoras}

El marco conceptual, en tanto, se seleccionó a partir de trabajos epistemológicos incluyendo diversos conceptos sobre el uso de especies de plantas exóticas invasoras (e.g. "neophytes", "antropophytes", "neoindigenophytes", "alien plants", "exotic plants", "introduced plants", "non-native plants" [Braun-Blanquet \& Pavillard, 1922; Holub \& Jirásek, 1967; Richardson et al. 2000; Colautti \& MacIsaac, 2004; Pyšek et al. 2004]), y de la utilidad de estos conceptos en la literatura local (e.g. Domínguez et al. 2006; Paula \& Labbé, 2012). En este trabajo se usan y describen las categorias sobre estatus de invasión de Pyšek et al. (2004) dado bajo estas definiciones, los cuales han mostrado ser de gran utilidad en manejo de especies en áreas protegidas: a) Plantas nativas: plantas que se han originado en un determinado lugar sin el involucramiento de humanos o que han arribado sin intervención intencional o no intencional por humanos desde el área en que ellas son nativas; b) Plantas exóticas: taxa de plantas en un área dada cuya presencia es debida a intervención humana intencional o no intencional, o que han arribado a un área sin la ayuda de personas desde un área en la que son exóticas; c) Plantas casuales: plantas exóticas que pueden reproducirse ocasionalmente fuera de los cultivos en un área, pero eventualmente mueren porque no forman poblaciones auto-sustentables y dependen de introducciones recurrentes para su persistencia; d) Plantas naturalizadas: plantas exóticas que mantienen poblaciones autosustentables por al menos 10 años sin intervención directa de personas, ya sea por reproducción sexual (semillas) o asexual (tubérculos, bulbos, fragmentos),
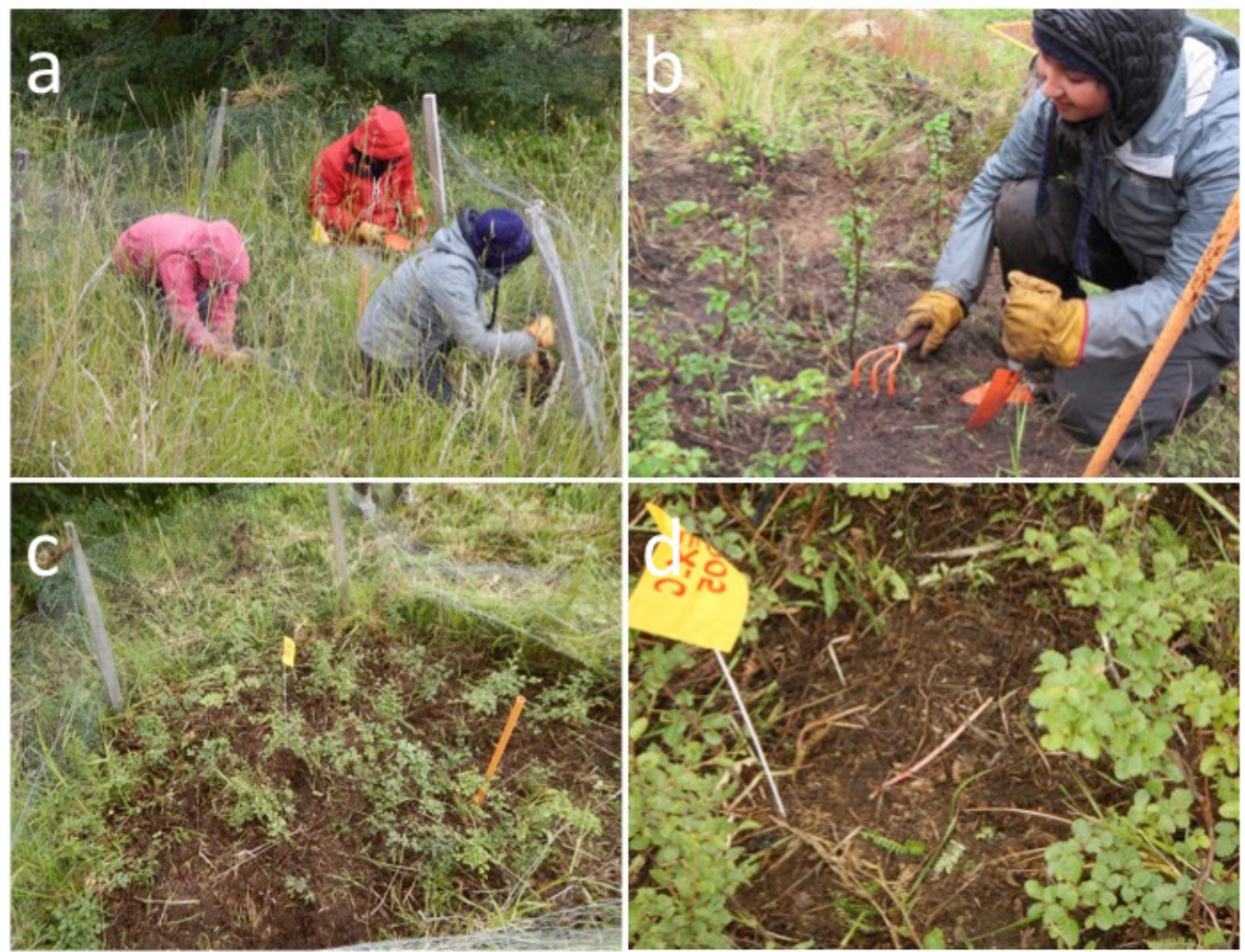

Fig. 3. Núcleos de plantación de Lenga en los que se realizó control de plantas invasoras mediante remoción mecánica de las partes aéreas de las plantas: a) Inicio de la remoción de plantas invasoras; b) Detalle del trabajo de remoción; c) Núcleo controlado, conteniendo sólo plántulas de Lenga; d) Detalle del núcleo controlado. 
capaces de desarrollarse independientemente; e) Plantas invasoras: plantas invasoras son un sub-grupo de las plantas naturalizadas que se reproducen eminentemente de forma sexual, a menudo con poblaciones muy numerosas y a distancia considerable de las plantas parentales, desarrollando así un potencial para expandirse sobre un gran área; f) Plantas transformadoras (edificantes): son un subgrupo de las plantas invasoras que cambian el carácter, condición o la naturaleza de los ecosistemas sobre una superficie importante; g) Plantas malezas: plantas que crecen en sitios donde no son deseadas para las cuales se ha detectado un impacto económico o ambiental de importancia (Pyšek et al. 2004) (Fig. 4).

\section{RESULTADOS}

Estado del arte sobre

las plantas exóticas invasoras

La literatura conteniendo información sobre especies de plantas exóticas en el Parque Nacional Torres del Paine es relativamente escasa (e.g. Skotsberg, 1916; Pisano, 1974; Arroyo et al. 1992; Domínguez et al. 2006) y ofrece tanto aproximaciones taxonómicas sobre los listados de especies ocurriendo en el área de estudio (e.g. Domínguez et al. 2006), así como información referente a los patrones distribucionales de las exóticas y los procesos que dan origen a la presencia de estas especies en ecosistemas naturales y perturbados (e.g. Dollenz, 1991). Sin embargo, es poca la información referida a explicar los mecanismos que permiten su permanencia en el tiempo y su eventual extensión (o disminución) en términos de abundancias y/o coberturas (e.g. Vidal \& Reif, 2011). Así, la determinación del proceso de invasión biológica en la literatura local es un cuestionamiento científico incipiente, que requiere de una evaluación tanto observacional como experimental en el área de estudio. En éste sentido, la literatura relevante sobre especies de plantas exóticas pueden clasificarse desde dos puntos de vista: 1) literatura taxonómica, cuyos resultados son catálogos florísticos y; 2) literatura ecológica, que aproximan explicaciones sobre mecanismos de invasión incipientes o evidentes en Torres del Paine.

\section{Literatura taxonómica}

sobre plantas exóticas en TDP

Los trabajos pioneros sobre especies de plantas exóticas en Torres del Paine corresponden a los de Skottsberg (1916) y Pisano (1974), quienes listan algunas de las especies más importantes en comunidades naturales ocurriendo en el área

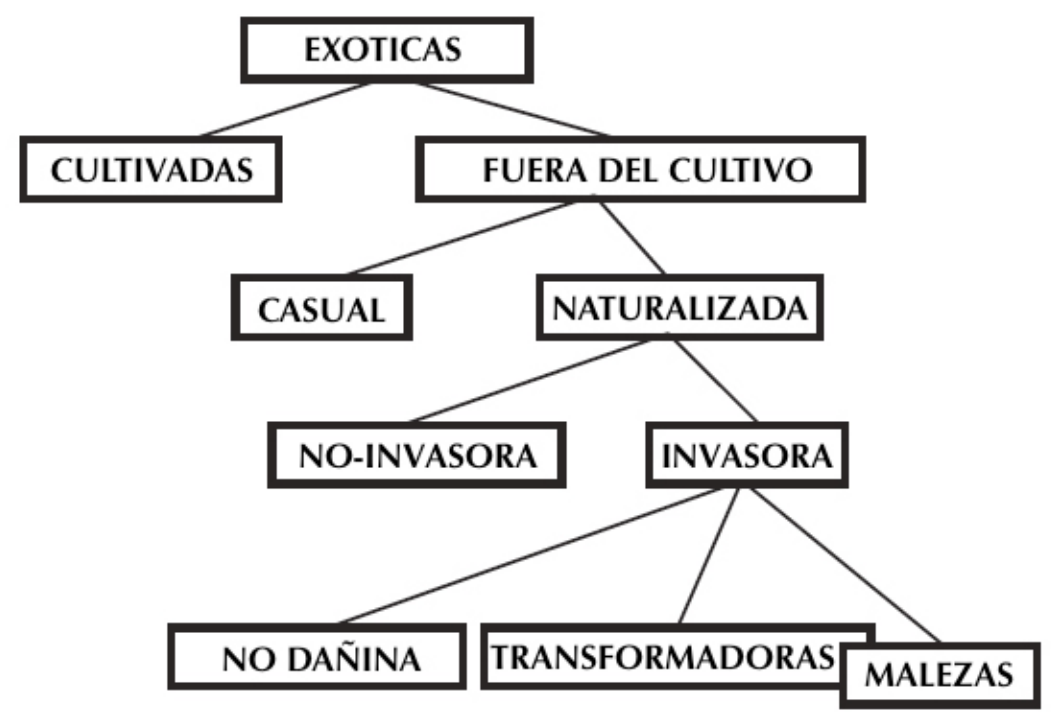

Fig. 4. Esquema jerárquico para la clasificación sugerida para las especies de plantas exóticas en el Parque Nacional Torres del Paine (adaptado de Pyšek et al. 2004). Nótese que las categorías de cada nivel son mutuamente excluyentes con la excepción de "malezas" y "transformadoras" que pueden sobreponerse. 
de estudio. El trabajo de Skottsberg (1916), correspondiente a una exhaustiva colecta de especies en el Sur de Patagonia, da cuenta de la presencia de sólo 2 exóticas: Cerastium arvense L. y Galium aparine L. Pisano (1974) en tanto, lista cuatro especies introducidas de origen Europeo: Cerastium arvense como componente de los Matorrales Mesófitos Pre-Andinos dominados por Escallonia rubra (Ruiz et Pavón) Pers. (Escallonietum rubrae deacuerdoala nomenclatura fitosociológica). Cerastium arvense también aparece en los bosques dominados por Lenga asociación denominada Nothofagetum pumiliae. Además, las especies Poa pratensis L. y Stellaria debilis d'Urb son nombradas en la monografía de Pisano como componentes del bosque bajo de Lenga, en asociaciones con fisonomías similares a las del Escallonietum rubrae. El trabajo de Pisano (1974), en este sentido, corresponde al mismo tiempo a la primera aproximación ecológica y taxonómica registrada para el área de estudio. Sin embargo, el objetivo de Pisano (1974) fue obtener la "determinación objetiva de la composición, extensión y distribución de las asociaciones vegetales" (sensu lato vegetación potencial [Dice, 1952]), lo que implicó excluir información referente a las comunidades antropogénicas y las especies que las componían debido, según el autor, "a la gran variabilidad encontrada en las características de la vegetación, resultantes de la diversidad de factores físicos, como a efectos del fuego y sobrepastoreo". De este modo, información importante sobre la presencia de las especies exóticas en tiempos relativamente antiguos, es obviada $y$ reaparece lustros más tarde, cuando el tema de las invasiones biológicas (sensu Lonsdale, 1999) recobra importancia.

Arroyo et al. (1992) documentan una exhaustiva prospección de plantas altoandinas, usualmente localizadas en hábitats sobre los 700 $\mathrm{m}$ de elevación que se corresponden al desierto andino (Pisano, 1974), por sobre el límite arbóreo. Esta flora comprende la presencia de 179 especies de las cuales sólo 2 (Rumex acetosella L. y Poa pratensis) son especies exóticas. Los autores no entregan antecedentes sobre la significancia ecológica de estas especies en sitios tan extremos como los ecosistemas alto-andinos.

El trabajo taxonómico más exhaustivo sobre las especies de plantas exóticas corresponde a la revisión elaborada por Domínguez et al. (2006), quienes registran la presencia de 85 especies de plantas introducidas en el área de estudio. El estudio es uno de los primeros en entronizar las invasiones biológicas como uno de los aspectos más importantes para ser analizados con fines de conservación en Torres del Paine y elaboran una exhaustiva revisión literaria sobre las especies documentadas para el parque, que complementada por prospecciones de campo que añaden 61 registros nuevos. El estudio recopila información importante sobre algunos rasgos funcionales de las especies como las formas de vida, el origen fitogeográfico y el estatus de invasión, significando un importante avance en la comprensión del fenómeno de las invasiones biológicas y la relevancia de éste para la conservación de la biota local. Sin embargo, aunque las especies del listado están categorizadas de acuerdo a niveles de invasibilidad (sensu Pyšek et al. 2004), el estudio no aporta con información que soporte el estatus de invasiones (e.g. información empírica sobre abundancias relativas de las especies), por lo que las categorías asignadas a cada especie de planta exótica, es un resultado que debe examinarse con cautela. Por ejemplo, la especie Aira caryophyllea L., es considerada meramente una especie "naturalizada" por los autores, implicando que las poblaciones de la especie no necesariamente invaden ecosistemas naturales, semi-naturales o antropogénicos (Pyšek et al. 2004). La especie, sin embargo ha sido registrada con altos niveles de cobertura en sitios afectados por incendios (e.g. Dollenz e Ivanovic, 1996; Paula \& Labbé, 2012). Dollenz e Ivanovic (1996) muestran cómo la especie registró coberturas paulatinamente incrementadas en sitios incendiados, desde $42 \%$ a $80 \%$ de cobertura tras 1 y 2 años postperturbación, respectivamente, mientras que en lugares de referencia, no incendiados, esta especie apenas estaba presente con un 3\% de cobertura. Este resultado muestra que las invasiones biológicas son contexto-dependientes y da señal sobre la importancia de evaluar los patrones de invasión de las especies en función del tipo, fecha y frecuencia de la perturbación, en vez de su categorización a priori como lo hacen Domínguez et al. (2004) careciendo de información empírica contrastable. 
Otros trabajos más contemporáneos también contribuyen a los catálogos florísticos con nuevas especies exóticas, no reportadas previamente. Clausen et al. (2006) por ejemplo, clasifican y describen los humedales en Torres del Paine y registran especies presentes en humedales localmente conocidos como vegas. Entre ellas, Potentilla anserina (L.) Rydb. representa una nueva contribución a la flora exótica, evidenciando que incluso los humedales presentan disrupciones antropogénicas que determinan cambios en su composición florística. Vidal (2007) en tanto, reporta nuevas especies de plantas exóticas como Oenothera stricta Ledeb. ex Link y Trifolium pratense L., sin ahondar en las características ecológicas de los hábitats en los que estas especies se hallaron, ni sobre las posibles causas de introducción.

\section{Literatura ecológica sobre plantas exóticas en TDP}

La literatura ecológica está representada por un número de trabajos relativamente importante y que, a diferencia de la literatura taxonómica sintetizada previamente, aporta con información relevante sobre los mecanismos involucrados en los procesos de invasión biológica en Torres del Paine.

Dos trabajos clásicos pueden ser considerados como pioneros sobre las invasiones biológicas en el área de estudio: El primer estudio, concerniente a evaluar la capacidad de colonización de Rumex acetosella en comunidades perturbadas por incendios en Torres del Paine (Dollenz, 1991), es una aproximación precursora sobre los rasgos funcionales de la especie que la habilitan para colonizar rápidamente los suelos denudados postperturbación. El autor evaluó la capacidad de colonización de esta especie tras el evento del incendio provocado por un turista Japonés en el año 1985. Rumex acetosella ha sido descrita por numerosos estudios en el Parque Nacional como una de las especies con mayor capacidad de invasión (e.g. Mansilla, 2007; Paula \& Labbé, 2012; Vidal \& Reif, 2011; Vidal \& Bauk, 2014), por lo que este estudio puede considerarse como visionario para su tiempo y muy influyente hoy en día para los planes de restauración. En particular, el autor discute cómo las características reproductivas sexuales (e.g. tamaño y peso de la semilla, producción de semillas por individuo) y asexuales (forma de vida, clonación subterránea por medio de estolones) de la especie ayudan a explicar su capacidad de "colonización" (hoy día el concepto sería "invasión") en espacios abiertos por incendios severos. Adicionalmente, la especie no sería palatable para los principales microherbívoros del área (e.g. liebre [Lepus Europaeus Pallas 1778] y el caiquén [Chloephaga picta Gmelin 1789]), por lo que escaparía a la presión por herbivoría (Dollenz, 1991). El segundo estudio de importancia para entender la naturaleza de las invasiones en Torres del Paine fue desarrollado por Dollenz \& Ivanovic (1996), quienes describieron el proceso sucesional en diferentes escalas espaciotemporales en un pastizal en el área de estudio, particularmente en el sector conocido como Pampa Grey. Los autores discuten cómo los tipos de propágulos disponibles inmediatamente después de la perturbación, especialmente los de especies con síndromes de dispersión anemócoros pueden tener mayores habilidades para la colonización de suelos desnudos. En particular, Rumex acetosella y Aira caryophyllea, ambas especies exóticas, fueron halladas con valores de cobertura más importantes en sitios afectados por el fuego, uno y dos años después de ocurrida la perturbación. Otras especies exóticas como Taraxacum officinale G. Weber y Myosotis discolor Pers. ex Murray son especies que aparecen en sitios incendiados y que no se encontraron en los sitios no incendiados de referencia (Dollenz \& Ivanovic, 1996).

Más tarde, Vidal (2005) estudió la influencia de los senderos de caminata y cabalgata de Torres del Paine sobre la distribución espacial de las especies de plantas exóticas, bajo el supuesto de que dichos senderos actúan como corredores para la introducción de propágulos de plantas exóticas en diferentes comunidades vegetacionales. El estudio reportó la presencia de 38 especies de plantas exóticas adyacentes a senderos, y una correlación inversa entre el número de especies exóticas y la distancia al borde de los senderos $(\rho=-0,529 ; \quad p<0,05 ; n=140 ;$ correlación no paramétrica de Spearman), sugiriendo que los senderos actúan como corredores de introducción para las especies de plantas exóticas e infestación de comunidades naturales. Esta tendencia general fue 
también significativa en los matorrales mesofíticos $(\rho=-0,500 ; p<0.05 ; n=140)$ recorridos por el sendero "Las Torres - Los Cuernos". Las especies de plantas exóticas mas importantes en términos de abundancia fueron Hypochoeris radicata L., Aira caryophyllea y Rumex acetosella, con la anemocoría (dispersión por viento), como el síndrome de dispersión más recurrente entre las especies exóticas reportadas (Vidal, 2005). El autor especulaba entonces sobre la fuerte influencia que podían tener estas especies frente a cambios ambientales, situación que quedó evidenciada después del evento de incendio del 2005, que significó en algunas comunidades incendiadas adyacentes a los senderos, una invasión masiva de especies exóticas (Mansilla et al. 2007).

Mansilla (2007) desarrolló un estudio que muestra los efectos del incendio del 2005 sobre una comunidad dominada por una planta leñosa endémica del sur de Sudamérica, la mata negra (Mulguraea tridens [Lag.] O`Leary \& P. Peralta [sinónimos Junellia tridens y Verbena tridens]). El incendio fue originado por un turista y destruyó ecosistemas presentes principalmente en la sección Oriente del Parque Nacional. La especie dominante forma extensos matorrales en la Estepa Patagónica al suroriente del Parque Nacional Torres del Paine, denominados en la literatura fitosociológica como el Matorral Verbeno-Junielletum tridentis Roig (Pisano, 1974; Roig et al., 1985; Roig, 1998). Mansilla (2007) muestra los efectos a corto plazo del incendio sobre la comunidad, por medio de la comparación directa de 10 rodales no incendiados y sus inmediatamente aledaños, incendiados. En lo referente a especies exóticas, el autor da cuenta de un leve, aparentemente no significativo incremento de la riqueza estas especies en los lugares incendiados, desde 12 a 15 especies en los sitios no incendiados $e$ incendiados, respectivamente (resultados descriptivos presentados sin pruebas estadísticas). Los valores de coberturas absolutos, sin embargo, permiten inferir que algunas especies fueron capaces de invadir sitios perturbados. Aira caryophyllea, por ejemplo, presentó uno de los valores más altos de cobertura para sitios incendiados con 9,3\%, mientras que Cerastium arvense mostró valores de 6,1\%. Otras exóticas, como Rumex acetosella y Plantago lanceolata L. mostraron valores de cobertura de $6,7 \%$ y
$5,3 \%$, respectivamente. Un re-cálculo de los datos del autor indican que las especies exóticas incrementaron en promedio desde $26 \%$ hasta $39 \%$ en cobertura desde los sitios no incendiados a los incendiados, respectivamente.

Posteriormente, Vidal \& Reif (2011) desarrollaron un estudio de caso para evaluar el efecto a corto plazo del incendio del año 2005 en la diversidad estructural y composicional de los bosques de lenga en el sector de Laguna Azul, Sierra Masle. El incendio, causado por un turista de nacionalidad Checa, provocó la destrucción de cerca de 17.000 hectáreas de diversos ecosistemas, de ellos unas 2.400 cubiertas por bosques de lenga (Navarro-Cerrillo et al. 2008). A través de una comparación directa entre rodales incendiados y rodales aledaños no incendiados, los autores encontraron que 4 años después del incendio, los sitios quemados estaban fuertemente invadidos por especies de plantas exóticas. La riqueza de exóticas en sitios de referencia no incendiados fue en promedio de $0,6 \pm 1,1$, mientras que los sitios incendiados tenían en promedio 11,9 \pm 4,2 especies, conformando el $44 \%$ de la riqueza total de especies en sitios incendiados (Vidal \& Reif, 2011). La mayoría de las especies exóticas en sitios invadidos fueron herbáceas forrajeras, cuya presencia en el Parque Nacional, especulan los autores, se debió probablemente a la influencia ganadera del área (Martinic, 1985) previa a su protección por el estado en 1956 (Vidal, 2012a). Otro hallazgo de importancia de este trabajo es la baja regeneración natural por plántulas de Lenga en sitios incendiados, que podría deberse a la interacción negativa (=competencia) de las plantas exóticas sobre la regeneración (Vidal \& Reif, 2011).

Vidal (2012b) desarrolló un estudio para evaluar la interacción entre incendios antiguos (ocurridos alrededor de 1940) y la ganadería actual sobre atributos de composición y función de la vegetación del sotobosque en bosques de lenga en Torres del Paine. El estudio fue posible de realizar debido a la colindancia entre el Parque Nacional y la Estancia Cerro Paine, esta última enclavada dentro del área protegida. La Estancia Cerro Paine (4.400 hectáreas) es de uso ganadero conteniendo 130 caballos a lo largo del año (2.95 animales $\mathrm{km}^{-1}$ ) y 350 vacas (7.95 animales $\mathrm{km}^{-1}$ ), por lo que difiere del parque nacional en el uso de 
suelo. Adicionalmente, los sitios colindantes entre Estancia Cerro Paine y el Parque Nacional Torres del Paine fueron incendiados alrededor de 1940, por lo que fue posible establecer una comparación a lo largo de una estratificación con bosques no perturbados (en el parque nacional), bosques afectados por ganadería (en Cerro Paine), bosques afectados sólo por fuego (Parque Nacional) y bosques afectados por ganadería más fuego (en Cerro Paine). El estudio muestra efectos sinérgicos de la ganadería y los incendios en la riqueza total de especies de plantas, pero un efecto exclusivo de la ganadería (no del fuego) en el establecimiento de especies exóticas. Dicho de otro modo, los sitios afectados por incendios antiguos (>70 años) tienden a recuperar con el tiempo la composición de especies nativas. Este resultado es relevante en restauración ecológica puesto que da cuenta de que bajo ciertas condiciones, la invasión biológica en bosques es un fenómeno que no perdura más que algunas décadas. Sin embargo, cabe destacar que aunque los bosques incendiados recuperan la composición de especies nativas, la identidad de las especies difiere de las de los bosques de referencia no intervenidos. Esta situación se daría principalmente al no existir árboles dominantes. Esto significa que al menos a una escala de rodal, existe baja o nula regeneración de Lenga en sitios incendiados, incluso tras décadas de ocurrida la perturbación (Vidal, 2012b). El autor especula que la ausencia de árboles semilleros podría explicar este aparente cambio en la trayectoria histórica (sensu SER, 2004) del ecosistema boscoso. También señala la importancia de estos árboles semilleros en la restauración pasiva, que como legados biológicos (sensu Foster et al. 1998), podrían asistir en la recuperación de funciones ecosistémicas post-perturbación (Vidal, 2012a).

Apenas unos meses después de extinguido el último incendio de la temporada 2011-2012, Paula \& Labbé (2012) elaboraron una innovadora aproximación para explorar preliminarmente los efectos del evento en la diversidad de plantas de la vegetación en diferentes ecosistemas del parque nacional, con énfasis en especies exóticas y el potencial de regeneración vegetativa de especies nativas y exóticas. El estudio muestra que muchas especies nativas leñosas tienen capacidad de regenerar vegetativamente después de los incendios: entre ellas Nothofagus antarctica (G. Forst.) Oerst., Chiliotrichum diffusum (G. Forster) Kuntze Discaria chacaye (G. Don) Tortosa, Ribes magellanicum Poiret, Berberis ilicifolia L. f., Embothrium coccineum J. R. Et. G. Forster, Berberis microphylla G. Forster, Gaultheria mucronata (L. f) Hook. Et Arn. y Escallonia sp. (posiblemente E. Rubra [Ruiz et. Pavón] Pers.), Berberis empetrifolia Lam y Senecio patagonicus Hook. et Arn. Esto mostraría el alto potencial de resiliencia de algunos ecosistemas australes al fuego. Algunas herbáceas también son capaces de rebrotar después de que el fuego destruyera parcial o totalmente sus tejidos aéreos. Entre estas últimas Arjona patagonica Hombron et Jacquinot, Armeria maritima (Miller) Willd., Acaena pinnatifida Ruiz et Pavón, Acaena ovalifolia Ruiz et Pavón, Poa pratensis, Plantago lanceolata, Festuca sp. (posiblemente F. gracillima Hook. f.), Valeriana carnosa Sm y Adesmia lotoides Hook. f, entre otras. La evaluación de Paula \& Labbé (2012) significa un importante avance en la comprensión del fuego como factor de perturbación de ecosistemas australes, dando cuenta de que muy probablemente, algunos ecosistemas tienen una rápida y positiva respuesta mediante rebrotes vegetativos de algunos sus componentes (i.e. poblaciones de especies), planteando la interrogante de si algunos ecosistemas tienen en efecto un origen post-incendio. Entre las especies exóticas que invaden y rebrotan con rapidez post-incendio, Paula \& Labbé (2012) encontraron que Poa pratensis, Plantago lanceolata, Polygonum aviculare L., Rumex acetosella, Cardus thoermeri Weinm., Achillea millefolium L., Dactylis glomerata L., Aira caryophyllea, Cerastium arvense, Agrostis capillaris L., Taraxacum officinale y Trifolium repens podrían considerarse invasoras no sólo por su capacidad de rebrote sino también por su potencial de colonizar suelos denudados mediante dispersión anemócora de sus propágulos (Vidal \& Reif, 2011). Una reinterpretación del marco conceptual propuesto por autores como Vidal \& Reif (2011) y Paula \& Labbé (2012) permiten asumir que la comprensión del fenómeno de las invasiones biológicas, sus mecanismos y su confrontación como problemática en restauración ecológica, pasa posiblemente por la evaluación y el análisis de los atributos en la historia de vida de las 
plantas, temática conocida en la literatura científica como los "rasgos funcionales de plantas" (en inglés "plant functional traits") (Cornelissen et al. 2003).

Los resultados del trabajo de Paula \& Labbé (2012) fueron extendidos a través de análisis experimentales sobre viabilidad $y$ germinación de semillas de plantas nativas y exóticas, y presentados en la tesis de Labbé (2013). El objetivo principal de la tesis consistió en determinar la regeneración sexual post-incendio de los ecosistemas Patagónicos. La autora evaluó experimentalmente la capacidad germinativa del banco de semillas en muestras de suelo extraídas en Torres del Paine, así como evaluó el efecto de diferentes intensidades de fuego (i.e. temperaturas) en la viabilidad y capacidad germinativa de semillas de 12 especies de plantas vasculares (9 nativas, 3 exóticas). Los resultados más relevantes muestran que la capacidad germinativa del banco de semillas en suelos incendiados, comparados con los suelos no incendiados, es significativamente baja. Algunos sitios representando a diferentes ecosistemas como Mirador del Salto (e.g matorral mesófito con Baccharis magellanica (Lam.) Pers. y Berberis microphylla), Angostura Río Paine (Bosque Caducifolio dominado por Nothofagus pumilio) y El Sarmiento (Matorral Xerófito dominado por Mulinum spinosum (Cav.) Pers. y Senecio patagonicus) presentaron valores extremadamente bajos de emergencias de plántulas. Importante también es destacar que las semillas de especies exóticas arrojaron los mayores porcentajes de germinación en suelos expuestos a altas temperaturas. Por ejemplo, a temperaturas de $90^{\circ}$ Celsius ninguna de las 5 especies nativas del experimento germinó, mientras que las especies exóticas como Dactylis glomerata, Rumex acetosella y Holcus lanatus L. lograron germinar tras exposiciones a $90^{\circ}$ Celsius por 10 minutos. Incluso, Holcus lanatus logró germinar a temperaturas después de estar expuestos a temperaturas de $120^{\circ}$ Celsius (Labbé, 2013). Contrariamente, semillas de especies nativas como Armeria maritima, Embothrium coccineum, Mulguraea tridens, Osmorhiza chilensis Hook. et Arn. y Senecio patagonicus lograron germinar a exposiciones de $60^{\circ}$ Celsius por 5 minutos, pero no a exposiciones de $90^{\circ}$ Celsius. Los resultados sugieren una baja resiliencia de los ecosistemas
Patagónicos al fuego, y una dependencia de los ecosistemas a fenómenos como el rebrote vegetativo de sus especies y a la dispersión de propágulos anemócoros para el restablecimiento de las especies nativas que redunde en la recuperación natural (i.e. restauración pasiva) de los ecosistemas. Alternativamente, los resultados muestran la gran capacidad invasora de las especies exóticas estudiadas, derivadas de su respuesta positiva a la exposición del fuego y a su capacidad adicional de reproducirse vegetativamente aun cuando su cuerpo vegetativo es parcialmente dañado por el fuego.

Vidal \& Bauk (2014) realizaron el primer análisis con implicancias directas en manejo y restauración ecológica de Torres del Paine en ecosistemas afectados por incendios. Este análisis se desarrolló en el contexto de la primera reforestación con fines de investigación científica y restauración ecológica en Torres del Paine. El plan fue ejecutado por la ONG AMA Torres del Paine bajo el título "Plan AMA Torres del Paine de restauración ecológica en bosques incendiados" (Vidal \& Bauk, 2014). Los autores, previo a la plantación de 20.000 lengas en sitios afectados por diferentes fechas de incendios, analizaron los patrones de distribución de especies exóticas y nativas en el sotobosque de bosques de lenga afectados por incendios ocurridos en 1985 (28 años post-perturbación), 2005 (8 años postperturbación) y 2011 (1 año post-perturbación). Los resultados, basados en el análisis de 83 micrositios muestran que los bosques afectados incluso por los incendios antiguos mantienen una alta diversidad de especies exóticas, con coberturas altas que podrían afectar los programas de reforestación en el plazo inmediato. Muchas de las especies exóticas son herbáceas con reproducción vegetativa (e.g. Holcus lanatus, Dactylis glomerata, Rumex acetosella), que invaden los micrositios despejados teniendo un alto potencial de competir con plántulas de lenga, por lo que podrían afectar seriamente la supervivencia y/o el crecimiento de éstas, se encuentren allí por medio de restauración pasiva o activa. Las recomendaciones de manejo de este reporte implican, entre otros, ejercer acciones periódicas de erradicación no sólo de las especies exóticas, sino de especies herbáceas nativas con la finalidad de eliminar la competencia a las plántulas de lenga. Por otra parte, recomiendan 
Riqueza de especies

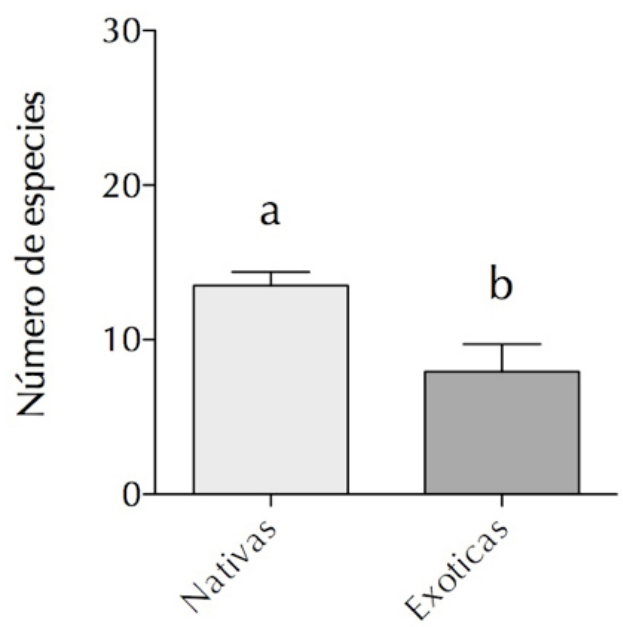

Cobertura de especies

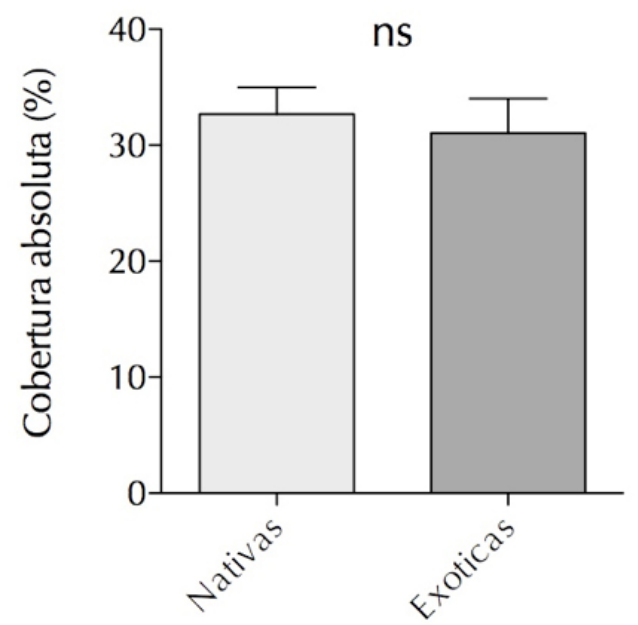

Fig. 5. Riqueza y cobertura de especies reportadas en la flora nativa y exótica en sitios afectados por el evento del incendio 2011-2012 en Torres del Paine. Diferentes letras indican diferencias estadísticamente significativas entre grupos (Mann-Whitney U-test, 95\%).

encarecidamente la selección de micrositios basados en legados biológicos. Estas estructuras pueden favorecer el micrositio para la supervivencia de plántulas plantadas mediante restauración activa, como ha demostrado el Plan de la ONG AMA Torres del Paine que ha logrado sitios hasta con 97\% de prendimiento 1 año después de ocurrida la plantación.
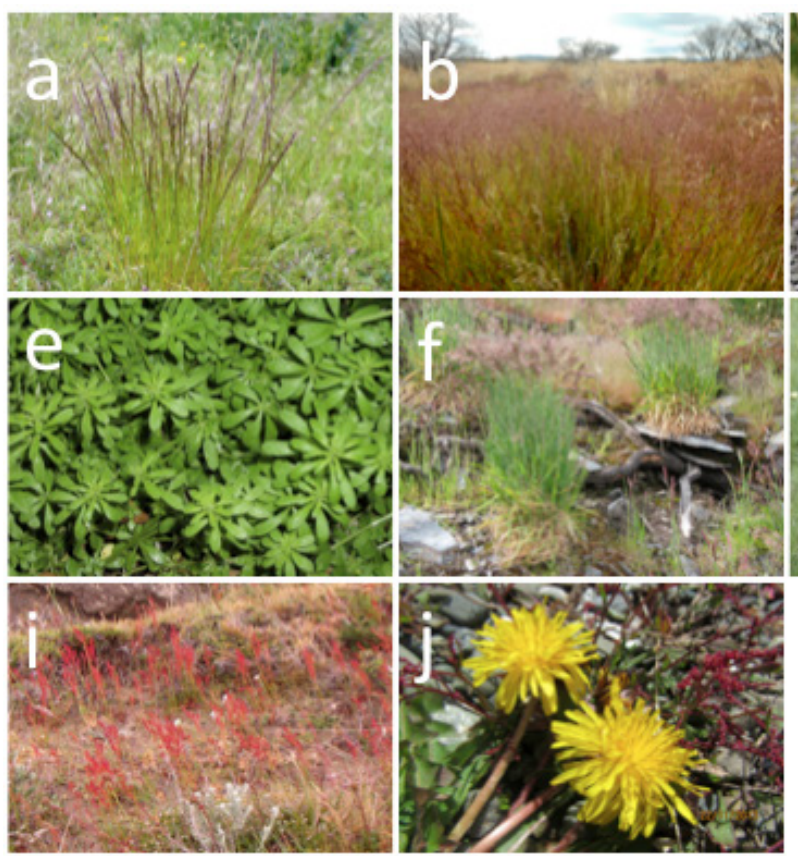

\section{Registro de exóticas y estatus de invasión}

Considerando el total de las unidades muestreales $(n=62)$, se registró un total de 150 especies de plantas vasculares, 108 (77\%) de ellas nativas y 34 (23\%) exóticas. En promedio se registraron $12,2( \pm 5,4)$ especies nativas por unidad de muestreo, mientras que las exóticas

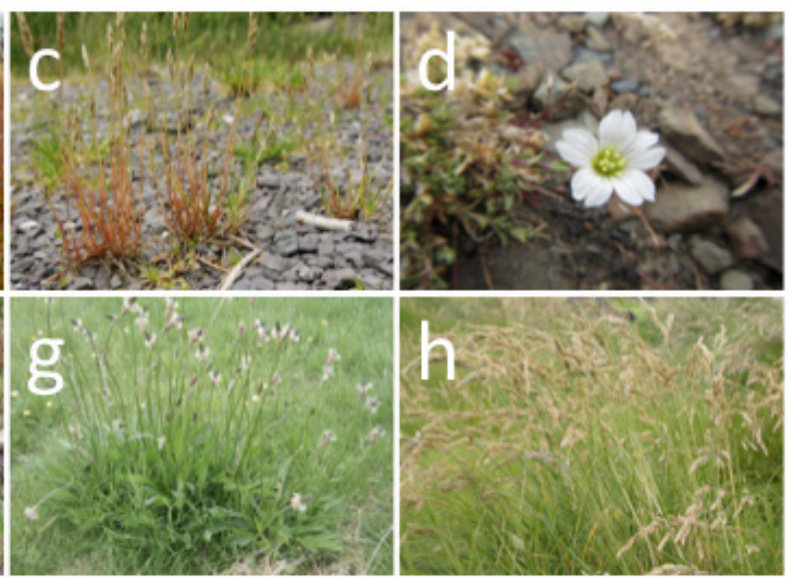

Fig. 6. Especies de plantas con mayor potencial de invasión postincendio en Torres del Paine: a) Grama de las boticas (Agropyron repens [L.] P. Beauv.); b) Chépica (Agrostis capillaris L.); c) Aira (Aira praecox L.); d) Cuernecita (Cerastium arvense L.); e) Lengua de Gato (Galium aparine L.); f) Pasto Miel (Holcus lanatus L.); g) Llantén (Plantago lanceolata L.); g) Heno (Poa pratensis L.); h) Vinagrillo (Rumex acetosella L.); i) Diente de León (Taraxacum officinale G. Weber ex Wigg.). 
Supervivencia de plántulas

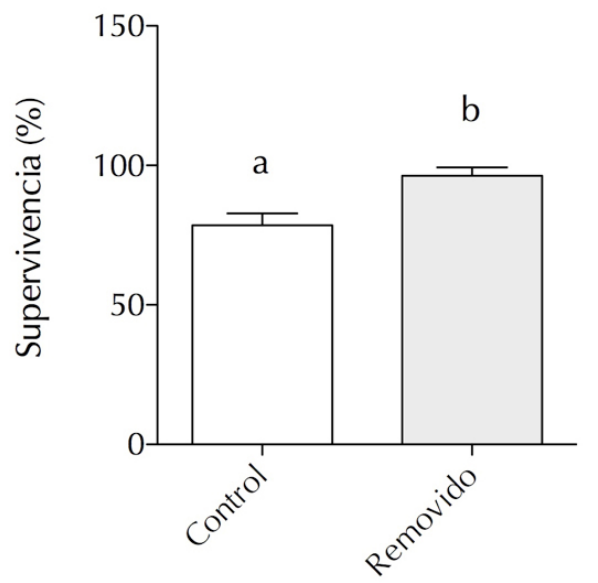

Fig. 7. Supervivencia de plántulas en núcleos de restauración ecológica del "Plan AMA Torres del Paine de Restauración de Bosques". La supervivencia de plántulas (\%) es comparada entre núcleos en los que se efectuó remoción de plantas herbáceas mediante extirpación manual, y núcleos no tratados (control), en los que no se removieron las especies de plantas invasoras (U-Mann Whitney test, 95\%).

promediaron $5,7( \pm 2,7)$ especies. En términos de cobertura absoluta, las especies nativas promediaron $32,6 \%( \pm 18,2)$ de la cobertura en las unidades de muestreo, mientras que las especies exóticas reportaron el 31,6\% ( $\pm 23,3$ DE) (Fig. 5).

Se seleccionaron 10 especies de plantas naturalizadas con carácter de invasoras en sitios afectados por el incendio del 2011-2012 (Fig. 6). Estas especies, consideradas como invasoras en muchos ecosistemas a lo largo de Chile (Ray et al. 2014) podrían tener un rol ecológico importante como transformadoras de la fisionomía del paisaje. El ranking es arbitrario pero basado en los grados de coberturas de las especies. 1) Vinagrillo (Rumex acetosella), familia Polygonaceae (cobertura relativa promedio 11,7); 2) Pasto miel (Holcus lanatus), familia Poaceae (cobertura relativa promedio 8,6); 3) Diente de León (Taraxacum officinale), familia Asteraceae (cobertura relativa promedio 2,8 ; 4) Cerastio (Cerastium arvense), familia Caryophyllaceae (cobertura relativa promedio 1,4); 5) Aira (Aira praecox), familia Poaceae (cobertura relativa promedio 2,1); Siete venas (Plantago lanceolata), familia Plantaginaceae (cobertura relativa promedio 2,8); Pasto Azul (Poa pratensis), familia Poaceae (cobertura relativa promedio 2,4 );
Agropyron (Agropyron repens), familia Poaceae (cobertura promedio 3,1); Hierba del chancho (Agrostis capillaris), familia Poaceae (cobertura promedio 0,9); 10) Erophila (Galium aparine), familia Rubiaceae (cobertura promedio 1,5). Estas especies aparecieron consistentemente a través de toda el área de estudio con valores relativos altos de cobertura, o dominaron ampliamente en sectores espacialmente localizados.

\section{Experiencias de control de especies exóticas invasoras}

La supervivencia en los núcleos sin control de invasoras tuvo una media de $78,5 \%$ de supervivencia (rango 72,7 - 87,5), mientras que los núcleos en los que se removieron las plantas tuvieron una supervivencia mayor de 96,3\% (rango 92,1 - 100), cuya diferencia estadística fue significativa $(p<0,05)$.

Se realizó adicionalmente, un cálculo dilatado para el costo monetario de control mecánico-manual de plantas invasoras en sitios de restauración ecológica activa como sigue: 1) Mano de obra: Se necesitaron 6 voluntarios que, divididos en cuadrillas de 2 personas, despejaron simultáneamente la vegetación presente en 3 núcleos de restauración de aproximadamente 4 $\mathrm{m}^{2}$ cada uno en un tiempo de aproximadamente 60 minutos. Los núcleos están distantes entre si dependiendo de la disponibilidad de legados biológicos en la superficie a restaurar, de manera que el acceso a los mismos demanda tiempos desiguales. Por lo tanto un grupo de 6 voluntarios podrá despejar la vegetación de los 40 núcleos en aproximadamente 13 horas de trabajo, lo que en la práctica corresponde a dos días, incluyendo traslados a las áreas de remoción de invasoras; 2) Se arrendó un vehículo para el traslado dentro del parque nacional de 6 personas a las áreas para el control de invasoras (CLP\$ 75.000); 3) El combustible necesario para el traslado de los voluntarios dentro del parque nacional ida y regreso desde los sitios de la ONG AMA Torres del Paine - Sitio de Restauración Lago Grey se traduce en $113 \mathrm{Km}$ de recorrido, con un vehículo de rendimiento de aproximadamente $9,8 \mathrm{Km} / \mathrm{Lt}$ que requiere $11,5 \mathrm{Lt}$ de combustible a un precio actual de aproximadamente CLP\$ 853 por cada Lt= 
CLP\$ 9.809*2 días de operación $\approx$ CLP\$ 20.000 (precio del combustible al 12 febrero 2013); 4) La alimentación para cada voluntario por la jornada completa aproximadamente CLP $\$ 10.000 \mathrm{c} / \mathrm{u}=$ CLP\$ 60.000*2 días de operación = 120.000; 5) Los gastos de materiales para trabajo (palas, overall, guantes, tijeras) son de aproximadamente CLP\$ CLP\$ 384.000 pata 6 voluntarios. De esta manera 6 voluntarios trabajando por 8 horas al día (incluyendo tiempos de traslados), serían capaces de controlar la flora invasora de un sitio como "Cuesta Margarita" de 40 núcleos (1,0 hectárea) en dos días, por un costo aproximado de CLP\$ 670.000. Esto demanda sin embargo, la participación de voluntarios, lo cual implica que los costos considerados en esta iniciativa solo incluyen gastos de logística y materiales. Hay que considerar sin embargo, que muy probablemente la operación de remoción de invasoras debe hacerse a principios de la estación de crecimiento vegetativo y a mediados de la misma, para asegurar que ellas no competirán con las plántulas de Lenga. Esto aumenta los costos de la operación al doble, con un valor final de CLP\$ 1.340.000 por hectárea.

\section{DISCUSIÓN}

El manejo ambiental sobre estas especies requiere también de la comprensión de dos conceptos claves en los planes de restauración ecológica: control y erradicación de especies. El control de especies, referido a las acciones que tiendan a limitar el crecimiento y expansión de las poblaciones en sitios en las que no son deseadas. Las acciones de control a menudo son espacialmente restringidas $e$ incluyen la remoción manual de los individuos vivos y tratamientos en los bancos de semillas (Mack et al. 2000). La erradicación en tanto, constituye las acciones que eliminen completamente a las poblaciones de la especie no deseada dentro de su ámbito de distribución no nativo, o en un caso menos estricto, su extirpación de un área geográfica amplia en la que su presencia no es deseada (e.g. un parque nacional) (Mack et al. 2000). Los resultados obtenidos sobre control de especies exóticas da cuenta de dos aproximaciones que pueden tener importantes implicancias en manejo: 1) La definición sobre invasiones biológicas, particularmente a la referencia sobre el concepto de plantas malezas (Pyšek et al. 2004) puede incorporar también a las especies de plantas nativas en micro-sitios (i.e. núcleos) en los que se está realizando reforestación y estas especies puedan entorpecer dichos planes mediante competencia o interacciones negativas con las plántulas de Lenga. Esto debido al complejo proceso que implica trabajar con voluntarios, a los cuales les resulta muy difícil distinguir las especies de plantas nativas y exóticas, especialmente en Torres del Paine donde todas las especies detectadas son herbáceas, de muy fácil confusión entre sí. Semejante ensayo realizado a una escala mayor requerirá del despeje de toda la competencia de las plantas que afecten el crecimiento de las plántulas de Lenga a lo menos durante el año inicial de crecimiento de las plantas de Lenga; Sin embargo dado el alto costo que implica su control, la opción alternativa sería plantar plántulas de Lenga de mayor altura en invernaderos (dos años o más), para asegurar que escaparán a la competencia de luz en altura con el estrato herbáceo. Con esta medida además se evitarían los costos de instalación y retiro de escudos protectores (shelters), práctica asentada en las reforestaciones del área de estudio y que representan problemas potenciales como contaminación y costos adicionales relativos al retiro de los escudos; 2) Las especies exóticas están ampliamente distribuidas en Torres del Paine. Su distribución temporal data probablemente de los tiempos de la colonización a principios de 1900 (Martinic, 1985; Vidal \& Reif, 2011) y su distribución espacial ha ido incrementando con los numerosos eventos de incendios ocurridos durante los últimos 30 años (Vidal, 2012a). Esta situación hace impracticable tanto desde el punto de vista económico como logístico la erradicación de estas especies en toda el área de estudio y por consecuencia, la propuesta sobre el control de estas especies estaría en: a) el monitoreo de invasiones incipientes por focos nuevos de invasión de especies recientemente registradas en el Parque Nacional y; b) El control de especies exóticas de larga data sólo en sitios con restauración activa, como en los núcleos de restauración del Plan AMA de Restauración de Bosques; c) El efecto ecológico de las especies exóticas debe estudiarse con más detalle en el futuro. Es posible que muchas especies cumplan con roles positivos post-fuego 
(e.g. evitar erosión del suelo), y cuya presencia sea sub-valorada para importantes funciones ecosistémicas; d) Finalmente, este estudio es sólo un reflejo temporal de un estado dinámico sujeto a un cambio temporalmente drástico. Las especies invasoras detectadas en este estudio son capaces de colonizar sitios severamente perturbados por incendios (e.g. suelo desnudo), pero no necesariamente son capaces de invadir ecosistemas naturales o seminaturales. En algunos casos es posible que estas especies sean solo oportunistas y disminuyan en abundancia cuando el espacio y los nutrientes liberados por el fuego desaparezcan, por lo que distinguir entre especies netamente colonizadoras-oportunistas $e$ invasoras requerirá de realizar estudios ulteriores. En este sentido, el monitoreo futuro de las estaciones de muestreo evaluadas y registradas en este estudio, es imperativo si se quiere conocer la naturaleza del fenómeno de las invasiones biológicas post-fuego en Torres del Paine. Decisiones basadas en las propuestas planteadas aquí, podrían ayudar a un efectivo y práctico manejo de áreas restauradas y con potencial de ser invadidas.

\section{AGRADECIMIENTOS}

Al proyecto Implementación de Acciones de Restauración Ecológica en Torres del Paine - Fase I, del Ministerio de Medio Ambiente y a los voluntarios de la ONG local, AMA Torres del Paine, quienes colaboraron con las actividades de terreno.

\section{LITERATURA CITADA}

Arroyo, M. T. K., von Bohlen, C. P, Cavieres, L., \& Marticorena, C. (1992). Survey of the alpine flora of Torres del Paine National Park, Chile. Gayana Botánica, 49, 47-70.

Cavieres, L. A., \& Fajardo, A. (2005). Browsing by guanaco (Lama guanicoe) on Nothofagus pumilio forest gaps in Tierra del Fuego, Chile. Forest Ecology and Management, 204, 237-248.

Clausen, J. C., Ortega, I. M., Glaude, C. M., Relyea, R. A., Garay G., \& Guineo, O. (2006). Classification of wetlands in a Patagonian National Park, Chile. Wetlands,
26, 217-229.

Chornesky, E., \& Randall, J. (2003). The threat of invasive alien species to biological diversity: setting a future course. Annals of the Missoury Botanical Garden, 90, 67-76.

Colautii, R. I., \& MacIsaac, H. (2004). A neutral terminology to define "invasive" species. Diversity and Distributions, 10, 135-141. Cornelissen, J. H. C., Lavorel, S., Garnier, E., Díaz, S., Buchmann, N., Gurvich, D. E., Reich, P. B., ter Steege, H., Morgan, D. H., van der Heijden, M. G. A., Pausas, J. G., \& Poorter, H. (2003). A handbook of protocols for standardized and easy measurement of plant functional traits worldwide. Australian Journal of Botany, 51, 335-380.

Couve, E., \& Vidal, C. (2007). Birds Torres del Paine ( $2^{\text {a }}$ edn.). Punta Arenas, Chile: Editorial Fantástico Sur.

De Cáceres, M., Legendre, P., \& Moretti, M. (2010). Improving indicator species analysis by combining groups of sites. Oikos, 119, 1674-1684.

Dice, L. R. (1952). Natural Communities (1 ${ }^{\text {rst }}$ edition). Michigan, USA: University of Michigan Press.

Dollenz, O. (1991). Capacidad de colonización de Rumex acetosella L. (Vinagrillo) en comunidades perturbadas. Anales del Instituto de la Patagonia, 20, 61-66.

Dollenz, O., \& Ivanovic, J. (1996). Sucesión secundaria en un pastizal incendiado en el Parque Nacional Torres del Paine", Magallanes, Chile. Anales del Instituto de la Patagonia, 24, 15-28.

Domínguez, E., Elvebakk, A., Marticorena, C., \& Pauchard, A. (2006). Plantas introducidas en el Parque Nacional Torres del Paine, Chile. Gayana Botánica, 63, 131-141.

Dufrene, M., Legendre, P. (1997). Species assemblages and indicator species: the need for a flexible asymmetrical approach. Ecological Monographs, 67, 345-366.

Foster, D. R., Knight, D. H., \& Franklin, J. F. (1998). Landscape patterns and legacies resulting from large, infrequent forest disturbances. Ecosystems, 1, 497-510.

García, M. D. (1998). Manual básico de literatura 
gris. El lado oscuro de la documentación. Asturias, España: Editorial Trea.

Gelbard, J. L., \& Belnap, J. (2003). Roads as conduits for exotic plant invasions in semiarid landscape. Conservation Biology, 17, 420-432.

Henríquez, J. M., Pisano, E., \& Marticorena, C. (1995). Catálogo de la flora vascular de Magallanes (XII Región), Chile. Anales del Instituto de la Patagonia, 23, 5-30.

Holub, J., \& Jirásek, V. (1967). Zur Vereinheitlichtung der Terminologie in der Phytogeopgraphie. Folia Geobotanica Phytotaxa, 2, 69-113.

Huber, U. M., \& Markgraf, V. (2003). European impact on fire regimes and vegetation dynamics at the steppe-forest ecotone of Southern Patagonia. The Holocene, 13, 567-579.

Iriarte, J. A., Johnson, W. E., \& Franklin W. L. (1991). Feeding ecology of the Patagonia puma in southernmost Chile. Revista Chilena de Historia Natural, 64, 145156.

Jaksic, F. M., Iriarte, J. A., \& Jiménez, J. E. (2002). The raptors of Torres del Paine National Park, Chile: biodiversity and conservation. Revista Chilena de Historia Natural, 75, 449-461.

Labbé, D. L. (2013). Efecto del fuego sobre la viabilidad y germinación de semillas en ecosistemas Patagónicos. Tesis de Ingeniero en Conservación de Recursos Naturales. Valdivia, Chile: Facultad de Ciencias Forestales y Recursos Naturales, Universidad Austral de Chile.

Lonsdale, W. M. (1999). Global patterns of plant invasions, and the concept of invasibility. Ecology, 80, 1522-1536.

Mack, R. N., Simberloff, D., Lonsdale, D. M., Evans, H., Clout, M., \& Bazzaz, F. (2000). Biotic invasions: Causes, epidemiology, global consequences and control. Ecological Applications, 10, 689-710.

Mansilla, H. (2007). La comunidad de Junellia tridens y los efectos de fuego en el Parque Nacional Torres del Paine, Patagonia, Chile. Tesis de Licenciatura en Ciencias Biológicas, Punta Arenas, Chile: Facultad de Ciencias, Universidad de Magallanes.

Marticorena, A. (2006). Revision of the genus Acaena (Rosaceae) in Chile. Annals of the Missouri Botanical Garden, 93, 412-454.

Martinic, M. B. (1985). La ocupación y el impacto del hombre sobre el territorio. En O. Boelcke, D. M. Moore, \& F. A. Roif (Eds.), Transecta botánica de la Patagonia Austral (pp. 95-104). Buenos Aires: CONICET-Instituto de la Patagonia-Royal Society.

Ministerio del Medio Ambiente (2014). Implementación de acciones de restauración ecológica del Parque Nacional Torres del Paine - Fase I. Informe final. Santiago, Chile: Ministerio del Medio Ambiente.

Moreno, P. I., Vilanova, I., Villa-Martínez, R., Garreaud, R. D., Rojas, M., \& De PolHolz, R. (2014). Southern Annular Modelike changes in southwestern Patagonia at centennial timescales over the last three millenia. Nature Communications, 5, 4375.

Moore, D. M. (1983). Flora of Tierra del Fuego. Missouri, USA: Missouri Botanical Garden.

Mueller-Dombois, D., \& Ellenberg, H. (1974). Aims and methods of vegetation ecology. New York, USA: John Wiley \& Sons.

Mooney, H. A., \& Hobbs, R. J. (2000). Invasive species in a changing world. Dordrecht, Holanda: Kluwer Academic Publishers.

Navarro-Cerrillo, R. M., Hayas, A., García-Ferrer, A., Hernández-Clemente, R., Duhalde, P. \& González, L. (2008). Caracterización de la situación posincendio en el área afectada por el incendio de 2005 en el Parque Nacional Torres del Paine (Chile) a partir de imágenes multiespectrales. Revista Chilena de Historia Natural, 81, 95-110.

Noss, R. F. (1990). Indicators for monitoring biodiversity: a hierarchical approach. Conservation Biology, 4, 355-364.

Ortega, I. M., \& Franklin, W. L. (1988). Feeding habitat utilization and preference by guanaco male groups in the Chilean Patagonia. Revista Chilena de Historia Natural, 61, 209-216.

Paula, S., \& Labbé, D. L. (2012). Evaluación de los impactos del incendio Olguín (PN Torres del Paine) en la vegetación. 
Valdivia, Chile: Instituto de Ciencias Ambientales y Evolutivas, Facultad de Ciencias, Universidad Austral de Chile.

Pisano, E. (1974). Estudio ecológico de la región continental sur del área andino-patagónica. II. Contribución a la fitogeografía de la zona del Parque Nacional Torres del Paine. Anales del Instituto de la Patagonia, 5, 59-104.

Pyšek, P., D. M. Richardson, M. Rejmánek, G. L. Webster, Williamson, M., \& Kirschner, J. (2004). Alien plants in checklists and floras: towards better communication between taxonomists and ecologists. Taxon, 53, 131-143.

Ray, C. A., Escobedo-Echeverría, V., Castro, S. A., \& Jaksic, F. (2014). Apéndice: Catálogo de la flora y fauna invasora en Chile continental $e$ insular oceánico. En F. M. Jaksic, \& S. A. Castro (Eds.), Invasiones Biológicas en Chile (pp. 496498). Santiago: Ediciones UC.

Richardson, D. M., Pyšek, P., Rejmánek, M., Barbour, M.G., Panetta, F. D., \& West, C. J. (2000). Naturalization and invasion of alien plants: concepts and definitions. Diversity and Distributions, 6, 93-107.

Rodríguez, R., \& Quezada, M. (2003). Fagaceae. En C. Marticorena, \& R. Rodríguez (Eds.), Flora de Chile (pp. 64-78). Concepción: Universidad de Concepción.

Roig, F. A., Anchorena, J., Dollenz, O., Faggi, A. M., \& Mendez, E. (1985). Las comunidades vegetales de la transecta botánica de la Patagonia Austral. En Boelcke, O., D. M. Moore, \& F. A. Roig (Eds.), Transecta Botánica de la Patagonia Austral (pp. 350-518). Buenos Aires: Consejo Nacional de Investigaciones Científicas y Técnicas, Instituto de la Patagonia, Royal Society.

Roig, F. A. (1998). La vegetación de la Patagonia. En M. Correa (Ed.), Flora Patagonica Tomo I (pp. 48-166). Buenos Aires: Instituto Nacional de Tecnología Agropecuaria.

SER, Society for Ecological Restoration OInternational Science and Policy Working group (2004). The SER International Primer on Ecological Restoration. Tucson, USA: Society for Ecological restoration
International.

Skottsberg, C. (1916). Die Vegetationsverhältnisse längs der Cordillera de los Andes S. von 41 $\mathrm{SBr}$. Ein Beitrag zur Kentniss der Vegetation in Chiloé, Westpatagonien und Feuerland. Vetenskapakademiens Handlingar, 56.

Sokal, R., \& Rohlf, J. (1981). Biometry (2nd edition). New York, USA: W. H. Freeman and Company.

Vidal, O. J. (2005). Flora exótica adyacente a senderos remotos en el Parque Nacional Torres del Paine (Magallanes, Chile). Tesis de licenciatura en ciencias biológicas. Valdivia, Chile: Facultad de Ciencias, Universidad Austral de Chile.

Vidal, O. J. (2007). Flora Torres del Paine field guide ( $2^{\text {nd }}$ edition). Punta Arenas, Chile: Editorial Fantástico Sur.

Vidal, O. J. (2012a). Torres del Paine, ecoturismo $e$ incendios forestales: perspectivas de investigación y manejo para una biodiversidad erosionada. Bosque Nativo, 50, 33-39.

Vidal, O. J. (2012b). Anthropogenic disturbances affecting southern beech (Nothofagus pumilio) forests in Torres del Paine Biosphere Reserve, southern Chilean Patagonia. Doctoral thesis. Freiburg, Germany: Faculty of Forests and Environmental Sciences, Albert-Ludwigs University in Freiburg.

Vidal, O. J., \& Reif, A. (2011). Effect of a touristignited wildfire on Nothofagus pumilio forests at Torres del Paine Biosphere Reserve, Chile (southern Patagonia). Bosque, 32, 64-76.

Vidal, O. J., San Martín, C., Mardones, S., Bauk, V., \& Vidal, C. F. (2012). The orchids of Torres del Paine Biosphere Reserve: the need for species monitoring and ecotourism planning for biodiversity conservation. Gayana Botánica, 69, 136-146.

Vidal, O. J., \& Bauk, V. (2014). Plan AMA Torres del Paine de restauración ecológica en bosques incendiados de Torres del Paine. Punta Arenas, Chile: Dirección de Vialidad MOP.

Villa-Martínez, R., \& Moreno, P. I. (2007). Pollen evidence for variations in the 
southern margin of the westerly winds in SW Patagonia over the last 12,600 years. Quaternary Research, 68, 400-409.

Wikum, D. A., \& Sahnholtzer, G. F. (1978).
Application of the Braun-Blanquet cover abundance scale for vegetation analysis in land development studies. Environmental Management, 2, 323-329.

\section{Anexo 1}

Especies de plantas vasculares exóticas registradas en sitios perturbados por el incendio del 2011-2012 en el Parque Nacional Torres del Paine. Se indica además el estatus de invasión de acuerdo a los valores de frecuencia y cobertura de la especie registrados en el muestreo de terreno (62 unidades muestreales). La especie es considerada maleza cuando interviene en los núcleos de restauración ecológica según nuestras observaciones, pero el concepto de transformadora es en ocasiones, puesto entre paréntesis debido a que su función ecológica es desconocida.

Achillea millefolium L. Milenrama. Especie poco frecuente en sitios incendiados, cobertura promedio baja $<1 \%$, frecuencia muy baja 1/62. Exótica, fuera de cultivo, naturalizada, no invasora. Agropyron repens Gaertn. Grama de las boticas. Especie frecuente y localmente abundante en el sector incendiado de Pampa Grey. Cobertura promedio $>5 \%$, frecuencia alta 24/62. Exótica, fuera de cultivo, naturalizada, invasora, maleza, transformadora (?). Agrostis capillaris L. Chépica. Especie frecuente en sitios incendiados, cobertura promedio $<5 \%$, frecuencia alta 23/62. Exótica, fuera de cultivo, naturalizada, invasora, maleza, transformadora (?). Aira praecox L. Aira. Especie frecuente en sitios incendiados, aunque con valores bajos de cobertura promedio $<2 \%$, frecuencia 22/62. Exótica, fuera de cultivo, naturalizada, invasora, no dañina (?). Aira caryophyllea L. Aira. Especie frecuente en sitios incendiados, aunque con valores bajos cobertura promedio $<1 \%$, frecuencia media 11/62. Exótica, fuera de cultivo, naturalizada, no invasora. Bromus hordeaceus L. Bromus. Especie poco frecuente en sitios incendiados, con valores bajos de cobertura promedio $<1 \%$, frecuencia muy baja 2/62. Exótica, fuera de cultivo, naturalizada, no invasora. Carduus nutans L. Cardo. Especie poco frecuente en sitios incendiados, con valores bajos de cobertura promedio $<1 \%$, frecuencia muy baja 1/62. Exótica, fuera de cultivo, naturalizada, no invasora. Cerastium arvense L. Cuernecita. Especie frecuente de encontrar en sitios incendiados, con valores de cobertura promedio $>1 \%$, frecuencia 33/62. Exótica, fuera de cultivo, naturalizada, invasora, no dañina (?). Cerastium fontanum Baumg. Cerastio, Cuernecita. Especie poco frecuente en sitios incendiados, con valores de cobertura promedio muy bajos $<1 \%$, frecuencia 5/62. Exótica, fuera de cultivo, naturalizada, no invasora. Cirsium vulgare (Savi) Ten. Cardo. Especie poco frecuente de observar en sitios incendiados, con valores de cobertura promedio muy bajos $<1 \%$, frecuencia 5/62. Exótica, fuera de cultivo, naturalizada, no invasora. Coronopus dydimus (L.) Sm. Cervellina. Especie muy poco frecuente de observar en sitios incendiados. Valores de cobertura promedio muy bajos $<1 \%$, frecuencia 1/62. Exótica, fuera de cultivo, naturalizada, no invasora. Crepis capillaris (L.) Wallr. Chicoria loquilla. Especie muy rara en los sitios incendiados. Valores promedios de cobertura muy bajos < $1 \%$, frecuencia $2 / 32$. Exótica, fuera de cultivo, naturalizada, no invasora. Dactylis glomerata L. Pasto ovillo. Especie muy rara de observar en sitios afectados por incendios recientes. Valores promedios de cobertura muy bajos $<1 \%$, frecuencia $1 / 62$. Exótica, fuera de cultivo, naturalizada, no invasora. Esta especie, sin embargo, presenta muy altos valores de cobertura en sitios afectados por incendios antiguos, lo que da cuenta del proceso observado de invasión es probablemente, incipiente. Digitalis purpurea L. Dedalera. Especie muy rara de observar en el área de estudio, pero localmente abundante en los sectores de Lago Grey - Refugio Grey, en lo que podría tratarse de una incipiente invasión. Valores de cobertura muy bajos $<1 \%$, frecuencia $3 / 62$. Exótica, fuera de cultivo, naturalizada, no invasora. Descurainia sophia L. Hierba de Sofía. Especie 
rara de observar a través del área de estudio pero localmente abundante en bosques incendiados del Sector Grey. Valores actuales de cobertura $<1 \%$, frecuencia 5/62. Podría tratarse de una invasión incipiente, pero nuestros resultados nos permiten catalogarla como exótica, fuera de cultivo, naturalizada, no invasora. Erodium cicutarium (L.) Lher ex Aiton. Alfilerillo. Especie rara de observar en sitios recientemente incendiados. Valores de cobertura $<1 \%$, frecuencia 1/62. Exótica, fuera de cultivo, naturalizada, no invasora. Erophylla verna (L.) Chevall. Especie efímera, difícil detección avanzada la estación primaveral. Rara en sitios incendiados, cobertura promedio $<1 \%$, frecuencia 7/62. Exótica, fuera de cultivo, naturalizada, no invasora. Galium aparine L. Especie localmente abundante en bosques incendiados del sector Grey. Cobertura promedio $>1 \%$, frecuencia 4/62 . Exótica, fuera de cultivo, naturalizada, invasora, no dañina. Holcus lanatus L. Frecuente en sitios incendiados, rizomatosa invasora de suelos denudados. Cobertura promedio $>5 \%$, frecuencia alta 29/62. Exótica, fuera de cultivo, naturalizada, invasora, maleza, transformdora (?). Hypochaeris radicata L. Especie común de observar en los sitios incendiados, pero con valores de cobertura muy bajos. Cobertura promedio $<1 \%$, frecuencia 26/62. Exótica, fuera de cultivo, naturalizada, no invasora. Myosotis arvensis (L.) Hill. No me olvides. Especie rara, poco frecuente. Valores de cobertura promedio $<1 \%$, frecuencia $8 / 62$. Exótica, fuera de cultivo, naturalizada, no invasora. Petrorhagia dubia (Raf.) G. López \& Romo. Rara de observar en sitios denudados recientemente por la acción del fuego. Cobertura promedio $<1 \%$, frecuencia $7 / 62$. Exótica, fuera de cultivo, naturalizada, no invasora. Plantago lanceolata L. Especie frecuente a traves de toda el área de estudio. Cobertura promedio
$>1 \%$, frecuencia 14/62. Exótica, fuera de cultivo, naturalizada, invasora, no dañina (?). Poa annua L. Pastito de invierno. Rara de observar. Cobertura promedio $<1 \%$, frecuencia $5 / 62$. Exótica, fuera de cultivo, naturalizada, no invasora. Poa pratensis L. Pasto Azul. Frecuente en el área de estudio. Cobertura promedio $>1 \%$, frecuencia 9/62. Exótica, fuera de cultivo, naturalizada, invasora, maleza, transformadora (?). Prunella vulgaris (Raf.) G. López \& Romo. Poco frecuente. Cobertura promedio $<1 \%, 4 / 62$. Exótica, fuera de cultivo, naturalizada, no invasora. Rumex acetosella L. Vinagrillo. Muy abundante a traves de toda el área de estudio. Cobertura $>10 \%$, frecuencia 38/62. Exótica, fuera de cultivo, naturalizada, invasora, transformadora, maleza (?). Sagina procumbens L. Poco abundante. Cobertura $<1 \%$, frecuencia 6/62. Exótica, fuera del cultivo, naturalizada, no invasora. Sonchus asper L. Poco abundante. Cobertura $<1 \%$, frecuencia $2 / 62$. Exótica, fuera del cultivo, naturalizada, no invasora. Stellaria media (L.) Vill. Poco abundante. Cobertura $<1 \%$, frecuencia $1 / 62$. Exótica, fuera de cultivo, naturalizada, no invasora. Taraxacum officinale G. Weber ex F.H. Wigg. Especie importante a través de toda el área denudada por incendios recientes. Cobertura $>2 \%$, frecuencia 32/62. Exótica, fuera de cultivo, naturalizada, invasora, maleza, transformadora (?). Trifolium dubium Sibth. Especie poco importante. Cobertura $<1 \%$, frecuencia 2/62. Exótica, fuera de cultivo, naturalizada, no invasora. Trifolium repens L. Especie poco importante. Cobertura $<1 \%$, frecuencia 4/62. Exótica, fuera de cultivo, naturalizada, no invasora. Veronica serphyllifolia L. Especie poco importante. Cobertura $<1 \%$, frecuencia 5/62. Exótica, fuera de cultivo, naturalizada, no invasoras. 


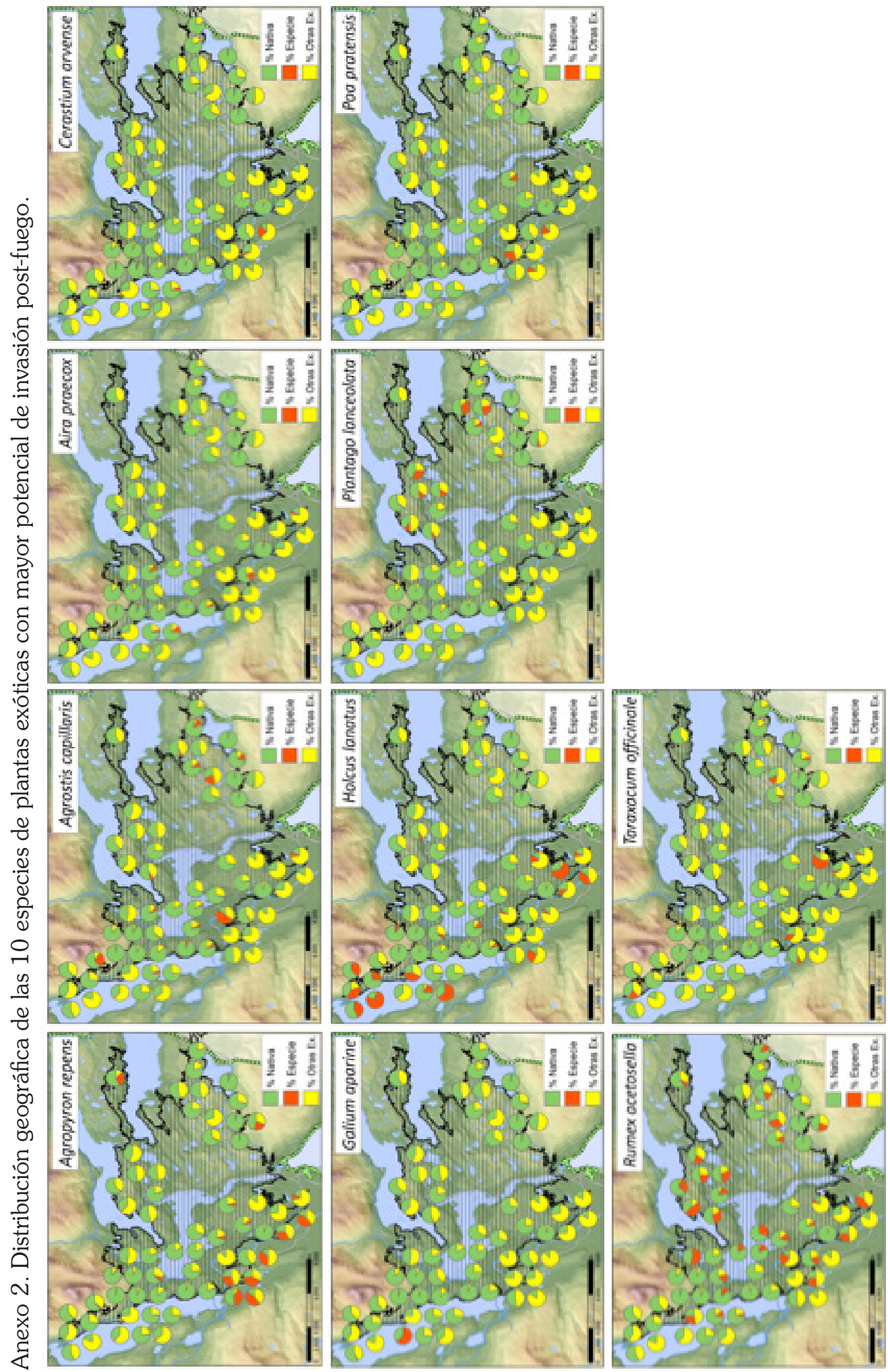

